\title{
Probing multi-phase outflows and AGN feedback in compact radio galaxies: the case of PKS B1934-63
}

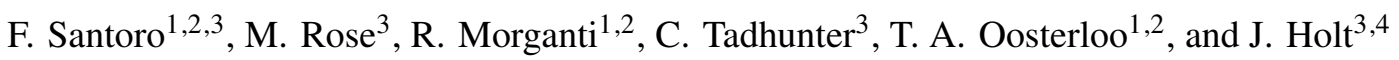 \\ 1 ASTRON, the Netherlands Institute for Radio Astronomy, PO 2, 7990 AA Dwingeloo, The Netherlands \\ 2 Kapteyn Astronomical Institute, University of Groningen, PO 800, 9700 AV Groningen, The Netherlands \\ 3 Department of Physics and Astronomy, University of Sheffield, Sheffield S3 7RH, UK \\ e-mail: f.santoro@sheffield.ac.uk \\ ${ }^{4}$ Leiden Observatory, Leiden University, PO Box 9513, 2300 RA Leiden, The Netherlands \\ e-mail: santoro@astro.rug.nl
}

Received 17 April 2018 / Accepted 22 June 2018

\begin{abstract}
Young radio AGN are pivotal for our understanding of many of the still-debated aspects of AGN feedback. In this paper we present a study of the interstellar medium (ISM) in the compact, peaked-spectrum radio galaxy PKS B1934-63 using X-shooter observations. Most of the warm ionized gas resides within a circum-nuclear disk with a radius of about $200 \mathrm{pc}$ that is likely to constitute the gas reservoir from which the central black hole feeds. On the other hand, we find a biconical outflow of warm ionized gas with an estimated radius of $59 \pm 12 \mathrm{pc}$. This matches the radial extent of the radio source and suggests that the outflow is jet driven. Thanks to the superior wavelength coverage of the data, we can estimate the density of the warm ionized gas using the trans-auroral line technique, and we find that the outflowing gas has remarkably high density, up to $\log n_{\mathrm{e}}\left(\mathrm{cm}^{-3}\right) \simeq 5.5$. The estimated mass outflow rate is low $\left(\dot{M}=10^{-3}-10^{-1} M_{\odot} \mathrm{yr}^{-1}\right)$, and the AGN feedback operates at relatively low efficiency $\left(\dot{E} / L_{\mathrm{bol}} \sim 10^{-4}-10^{-3} \%\right)$. In addition, optical and near-IR line ratios show that the expansion of the radio source drives fast shocks (with velocities $v_{\mathrm{s}} \gtrsim 500 \mathrm{~km} \mathrm{~s}^{-1}$ ) that ionize and accelerate the outflowing gas. At odds with the properties of other compact, peaked-spectrum radio sources hosting warm ionized gas outflows, we do not find signs of kinematically disturbed or outflowing gas in phases colder than the warm ionized gas. We argue that this is due to the young age of our source and thus to the recent nature of the AGN-ISM interaction, and suggest that cold gas forms within the outflowing material and the shock-ionized outflowing gas of PKS B1934-63 did not have enough time to cool down and accumulate in a colder phase. This scenario is also supported by the multi-phase outflows of other compact and young radio sources in the literature.
\end{abstract}

Key words. ISM: jets and outflows - evolution - galaxies: active - galaxies: evolution - galaxies individual: PKS B1934-63

\section{Introduction}

The interaction between the energy released by the central active nucleus (AGN) and the host galaxy's interstellar medium (ISM) is particularly prominent in compact and young radio galaxies, and one of the main manifestations of this interaction is visible in the jet-driven gas outflows that extend on scales of galaxy bulges (see, e.g., Fanti et al. 1990; Fanti \& Fanti 1994; Axon et al. 2000; O'Dea et al. 2002; Holt et al. 2006, 2008; Geréb et al. 2015a,b). In the context of galaxy evolution, the negative feedback effect that such outflows, and thus AGN, have on the host galaxy has a crucial role in explaining, for example, scaling relations between the central black hole $(\mathrm{BH})$ and its host galaxy properties (Silk \& Rees 1998; Fabian 1999; King 2003; Granato et al. 2004; Di Matteo et al. 2005) and the quenching of the star formation (Benson et al. 2003; Bower et al. 2006; Bongiorno et al. 2016) in massive early-type galaxies (ETG).

Compact and young radio galaxies are identified by the (small) size of their radio emission, and based on the properties of their radio spectra, are classified as compact steep spectrum (CSS) or as gigahertz peaked sources (GPS) (e.g., Giroletti \& Polatidis 2009; Murgia et al. 1999; Murgia 2003). Many compact radio galaxies show clear signs of the interaction between the expanding radio jets and the surrounding dense and multi-phase ISM, which slows down (or even prevents) the jet expansion (see
Orienti \& Dallacasa 2008; Callingham et al. 2015; Tingay et al. 2015, and reference therein), in line with simulation predictions (Bicknell et al. 1997; Wagner et al. 2012, 2016).

These newly born AGN inflating their radio lobes into the surrounding ISM give us the unique opportunity to study many aspects of so-called AGN feedback. In particular, they can help us to probe the efficiency of the AGN feedback in different gas phases, and even more, investigate the origin of the cold gas that is often observed in this harsh environment (see, e.g., Dasyra \& Combes 2012; Tadhunter et al. 2014; Oosterloo et al. 2017). Currently, the acceleration mechanism of outflows is uncertain, and these sources are ideal for probing the relevance that shocks have in accelerating and ionizing outflowing gas.

Even though their actual impact is still unclear, ionized gas outflows are commonly found in compact, young radio sources, and they show more extreme features than the outflows in extended radio sources (Holt et al. 2008). In the case of the warm ionized gas, one of the important parameters that contributes to the uncertainties in the estimate of the AGN feedback efficiency is the gas electron density $n_{\mathrm{e}}$ (see Tadhunter 2016; Harrison et al. 2018 , for a discussion). The classical line ratios used as density diagnostic, such as the $\left[\mathrm{S} \mathrm{II}_{\mathrm{I}}\right] \lambda 6717 / \lambda 6731 \AA$ ratio, give a reliable estimate only for low densities (i.e., $10^{2}<n_{\mathrm{e}}<10^{3.5} \mathrm{~cm}^{-3}$ ) and saturate in the high-density regime (see Osterbrock \& Ferland 
2006). These low densities might not reflect the actual gas properties, especially in the case of compact radio galaxies, and might result in incorrect values for the mass outflow rate and the AGN feedback efficiency. Holt et al. (2011) and Rose et al. (2018) make use of the technique based on the [S II] and [O II] trans-auroral lines and find that the gas electron density can reach values up to $n_{\mathrm{e}}=10^{4-5} \mathrm{~cm}^{-3}$ for outflowing gas.

The occurrence and effects that shocks have on the ISM is also an important, although poorly quantified, component of AGN-driven outflows. It is known that both fast radio jets/lobes and AGN winds are able to shock and accelerate the ambient ISM along their path (see, e.g., Couto et al. 2013, 2017). Evidence and/or indications of the presence of shocks have often been reported for compact radio sources, and they are usually connected to the expansion of the radio source within the ambient ISM. The main evidence for shocks comes from Hubble Space Telescope high-resolution imaging studies of the warm ionized gas (de Vries et al. 1997b; Axon et al. 2000; Batcheldor et al. 2007; Labiano 2008), which in some cases have been complemented by spectroscopic observations of highly broadened emission lines (Holt et al. 2008). In other cases, kinematically disturbed cooler ISM phases (neutral and molecular) have been found at the location of radio lobes, clearly indicating shock acceleration (e.g., Oosterloo et al. 2000, 2017; Morganti et al. 2013; Tadhunter et al. 2014). Mainly due to limitations in the observations, pure spectroscopic evidence of shock-ionized gas is sparse and much harder to find.

Finally, outflows of atomic ( $\mathrm{HI}$ ) and molecular (warm $\mathrm{H}_{2}$ and $\mathrm{CO})$ gas have been observed in compact steep-spectrum radio sources such as IC 5063 (Tadhunter et al. 2014; Morganti et al. 2015), PKS B1345+12 (Morganti et al. 2013; Dasyra \& Combes 2012) and 3C 305 (Morganti et al. 2005a). However, the origin of the cold outflowing gas it is still not clear in the context of AGN feedback. A scenario that is gaining consensus predicts that molecular gas forms in situ, in particular in the post-shock regions of the outflows, rather then surviving the AGN-ISM interaction and being gradually accelerated by entrainment. This is supported by the molecular gas observations of the compact radio source IC 5063 (see Tadhunter et al.2014; Morganti et al.2015) and by recent simulations by Richings \& Faucher-Giguere(2017), showing that cold gas can form in the first few $10^{5} \mathrm{yr}$ after the start of the AGNISM interaction. Compact radio sources, with their young age and multi-phase outflows, are ideal objects on which to test this scenario.

In this paper, we use spectroscopic observations of the compact radio source PKS B1934-63 to characterize the efficiency of the AGN feedback for the warm ionized gas phase and compare it to other classes of objects. We also study the presence or relevance of shocks using line ratio diagnostics, and investigate the multi-phase nature of the outflowing gas using the emission of the warm molecular gas. Finally, we perform a first attempt to test the scenario in which cold gas forms within the post-shock regions of outflows by combining our findings with previously published results for other compact radio sources.

The source PKS B1934-63 $(z=0.1824)$ is a powerful radio $\operatorname{AGN}\left(\mathrm{P}_{1.4 \mathrm{GHz}}=10^{27.2} \mathrm{~W} \mathrm{~Hz}^{-1}\right)$ classified as a GPS by de Vries et al. (1997a). It has often been considered as the archetypal GPS source: it is one of the closest and most powerful compact radio sources and was one of the first GPS to be discovered (Bolton et al. 1963). Very long baseline interferometry (VLBI) observations resolved the radio source into two components, likely representing the two radio lobes, separated by
$131.7 \pm 0.9 \mathrm{pc}$ (Ojha et al. 2004). The kinematic age of the radio source has been estimated to be $1.6 \times 10^{3} \mathrm{yr}$ by monitoring the lobe separation over a timescale of about $32 \mathrm{yr}$ (Ojha et al. 2004).

The host galaxy of PKS B1934-63, identified by Fosbury et al. (1987), is an ETG that is undergoing a merger with a companion galaxy located about $9 \mathrm{kpc}$ away. Optical and IR images revealed the fainter companion together with clear tidal features (Heckman et al. 1986; Inskip et al. 2010; Ramos Almeida et al. 2011). The optical polarimetry study of Tadhunter et al. (1994b) found polarized light consistent with scattered AGN light, or with nonthermal emission connected to the radio structure.

More recently, Holt et al. (2008) and Roche et al. (2016) studied the conditions of the warm ionized gas in the host galaxy using slit and integral field spectroscopy, respectively. Both studies reported a broad blueshifted component in the [O III] $\lambda 5007 \AA$ line profile representing outflowing gas, and hints of high gas densities (measured via the classical [S II] $\lambda 6717 / \lambda 6731 \AA$ line ratio). Moreover, Roche et al. (2016) found that the velocity gradient of the outflowing gas component is aligned with the radio jets, and suggested shocks as the mechanism that ionizes the warm ionized gas.

Here, we present long-slit spectroscopic observations of PKS B1934-63 obtained with the X-shooter instrument (Vernet et al. 2011) mounted at the VLT. We take advantage of the large wavelength coverage and of the good velocity resolution of the X-shooter data to study the outflowing gas and probe the warm ionized gas electron density via the trans-auroral line technique (Sect. 3). In addition, using the spectro-astrometry technique, we study the spatial extent of the different kinematical components of the ionized gas (Sect. 4). This allows us to obtain a better estimate of the mass outflow rate and of the efficiency of the AGN feedback (Sect. 5). We also investigate the ionization state of the warm ionized gas using line ratio diagnostic diagrams to probe for the presence of shocks within the outflowing material (Sect. 6). Finally, we study the kinematics of the warm molecular gas and link it to the kinematics of the warm ionized and atomic gas (Sect. 7).

Throughout this paper we assume the following cosmology: $\mathrm{H}_{0}=70 \mathrm{~km} \mathrm{~s}^{-1} \mathrm{Mpc}^{-1}, \Omega_{0}=0.28$, and $\Omega_{\lambda}=0.72$. At the redshift of PKS B1934-63, 1 arcsec $=3.091 \mathrm{kpc}$.

\section{Observations and data reduction}

Observations were carried out with X-shooter at the VLT/UT2 on July 1, 2011, in visitor mode and with a total exposure time of $75 \mathrm{~min}$ (i.e., $10 \times 450 \mathrm{~s}$ for the visual arm (VIS), $5 \times 900 \mathrm{~s}$ for the ultraviolet-blue arm (UVB), $15 \times 300 \mathrm{~s}$ for the near-IR (NIR)) arm. In order to facilitate sky subtraction, separate exposures were taken with the slit nodded off source. The instrument was used in SLIT mode with $1.6 \times 11$ arcsec slit for the UVB arm, $1.5 \times 11$ arcsec slit for the VIS arm and $1.5 \times 11$ arcsec slit for the NIR arm. The selected slit position angle (PA) was set to be 104 degrees (from north to east), close to the PA of the source's radio axis (i.e., 90 degrees, Tzioumis et al. 1989) and including both the PKS B1934-63 host galaxy and the fainter merging or interacting companion.

To estimate the seeing, we used three sets of acquisition images taken during the observations, measuring the profiles of seven stars in the images. For each star we extracted a spatial profile, using a mock slit with the same size of the slit used for the actual observations, and we fit it with a Gaussian function. 


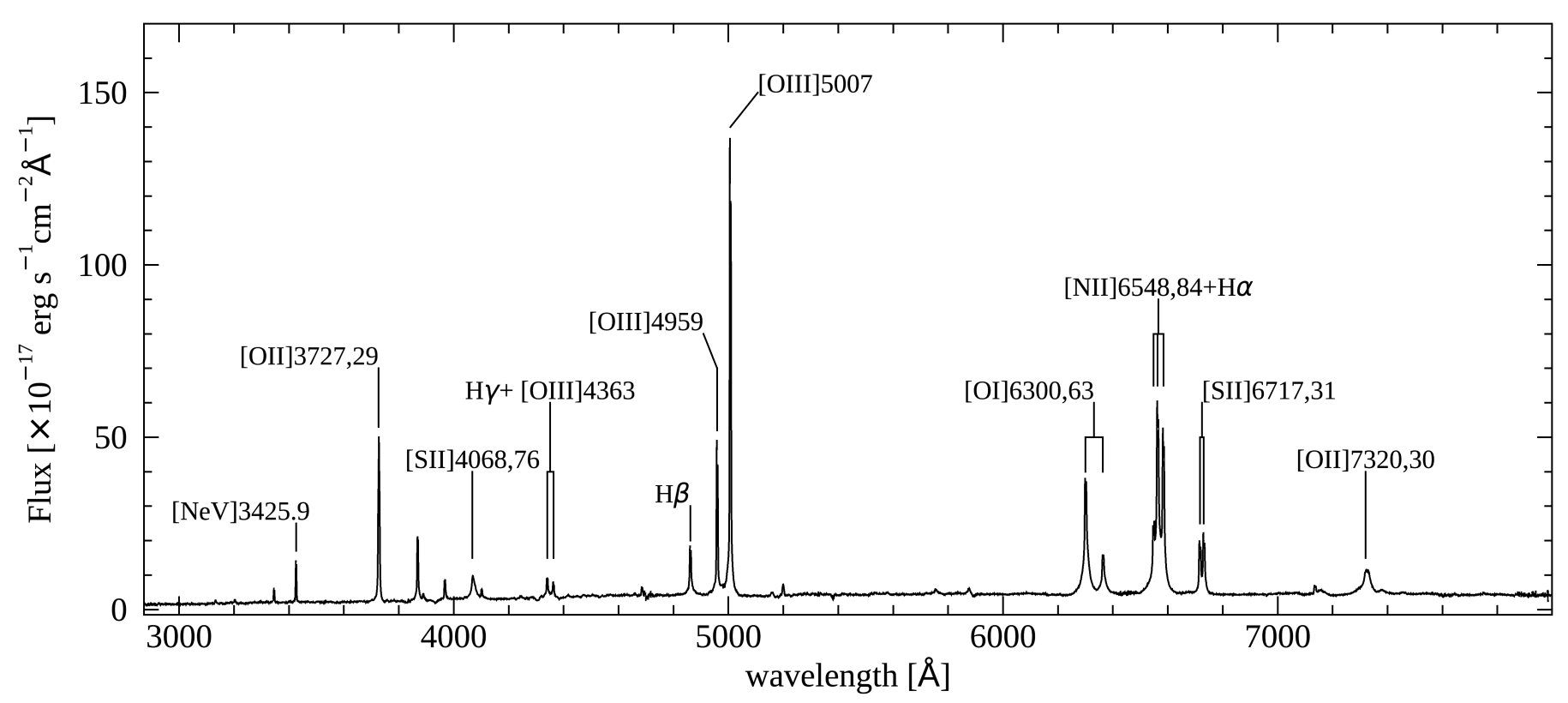

Fig. 1. UVB+VIS nuclear spectrum of PKS B1934-63. The main emission lines are indicated. Wavelengths are plotted in $\AA$, and the flux scale is given in units of $10^{-17} \mathrm{erg} \mathrm{s}^{-1} \mathrm{~cm}^{-2} \AA^{-1}$.

The seeing was then estimated taking the average full width at half-maximum (FWHM) of the fitted 1D profiles. In this way, we obtained a seeing value of $0.97 \pm 0.06$ arcsec that takes into account the integration of the seeing profile across the slit in the dispersion direction. The uncertainty in the seeing is the standard error of all the seeing values extracted from the acquisition images.

Standard data reduction was performed using the ESO REFLEX workflow and included bias subtraction, flat fielding, and flux calibration. For each arm, we applied second-order calibrations to the final pipeline products. Residual hot and bad pixels were removed using FIGARO BCCLEAN. Sky subtraction was performed on the slit spectra by extracting an average sky spectrum from the regions of the slit devoid of sources (i.e., the top and bottom part of the slit). We also performed a telluric absorption-line correction using the integrated spectrum of a standard star observed during the same night.

We derived the average accuracy of the wavelength calibration and the average instrumental width by measuring the line centers and FWHM of sky emission lines, respectively. We found that the wavelength calibration accuracy is $20 \mathrm{~km} \mathrm{~s}^{-1}$, $5 \mathrm{~km} \mathrm{~s}^{-1}$, and $3 \mathrm{~km} \mathrm{~s}^{-1}$, while the instrumental width is $90 \mathrm{~km} \mathrm{~s}^{-1}, 60 \mathrm{~km} \mathrm{~s}^{-1}$, and $90 \mathrm{~km} \mathrm{~s}^{-1}$ for the UVB, VIS, and NIR arms, respectively. The relative flux calibration accuracy was estimated to be $10 \%$ taking into account the flux variations of the source calibrated using three different standard stars from the same night. Considering that the radio source is $42.6 \pm 0.3$ mas in diameter (i.e., spatially unresolved by the current observations), we used the estimated seeing to set the aperture size and extract the nuclear spectrum of PKS B1934-63 (see Fig. 1).

\section{Data analysis and results}

The nuclear spectrum of PKS B1934-63 (shown in Fig. 1 for the UVB and VIS bands) is extremely rich in emission lines with complex line profiles. The spectrum shows typical features of an high-excitation radio source (HERG, Best \& Heckman
2012) and additional strong low-ionization lines such as the [O I], [O $\left.\mathrm{O}_{\mathrm{II}}\right]$, and [S $\left.\mathrm{II}\right]$ lines. We also detect absorption features for the $\mathrm{Mg}$ II $\lambda \lambda 2796,2804 \AA$ in the UVB, and $\mathrm{H}_{2}$ emission lines in the NIR. We find that the strongest emission lines show broad wings and are double peaked. This is discussed in more detail in Sect. 3.2 together with the line modeling.

\subsection{Redshift and stellar population modeling}

Deriving an accurate value for the redshift of the galaxy is essential for the determination of the velocity of any outflowing gas component. The available estimate of the host galaxy systemic velocity is based on bright AGN emission lines (Holt et al. 2008). These lines are often affected by the complex kinematics of the ionized gas, leading to uncertainties in the derived systemic velocity (Tadhunter et al. 2001; Comerford et al. 2009).

With our current data we were able to estimate the systemic redshift of the galaxy using the Ca II $\mathrm{K}$ stellar absorption (part of the Ca II $\lambda \lambda 3933,68 \AA$ doublet), which is free from the contamination of gas emission lines. We fit this line with a Lorentzian function and obtained a redshift $z=0.18240 \pm$ 0.00013 (the uncertainty on the redshift corresponds to about $\left.40 \mathrm{~km} \mathrm{~s}^{-1}\right)$.

However, the CaII K stellar absorption can potentially include absorption due to the ISM of the host galaxy. To verify that this did not have a significant impact on our redshift estimate, we used the ISM absorption lines of the Mg II $\lambda \lambda 2795,2802 \AA$ doublet. We found that the width of the $\mathrm{Mg}_{\text {II }}$ absorption lines was significantly lower than that of the Ca II K absorption (about seven times lower) and their velocity shift was compatible, within the errors, with the systemic velocity derived from the $\mathrm{Ca}$ II $\mathrm{K}$ absorption (see Appendix A for the details on the fitting procedure). Therefore, we conclude that the $\mathrm{Ca}$ II $\mathrm{K}$ absorption line profile is mainly related to stellar absorption and that our estimate of the redshift is robust.

We modeled the stellar population in the nuclear spectrum of the host galaxy using STARLIGHT (version 04, 
Cid Fernandes et al. 2005) and masking all the emission lines. With the aim of finding a simple model to fit the continuum emission, we used stellar templates with solar metallicity provided by STARLIGHT (Bruzual \& Charlot 2003) to model the stellar light. Given that Tadhunter et al. (1994b) detected scattered light from the central AGN, we also introduced a power law $\left(\mathrm{F}_{\lambda}=\lambda^{\alpha}\right)$ in our model to take this into account. The best-fit model of the galaxy continuum was chosen based on $\chi^{2}$ statistics and residual analysis. It includes a $2.5 \mathrm{Gyr}$ old stellar population and a power law with spectral index $\alpha=-0.1$. The redshift derived from the stellar population fit procedure is in line with our redshift estimate using the $\mathrm{Ca}$ II $\mathrm{K}$ absorption line. Our best-fit model is shown in Fig. A.1 and was subtracted from the nuclear spectrum of PKS B1934-63 before we modeled the gas emission lines.

\subsection{Kinematic model}

To obtain a reference model for the forbidden emission lines of the ionized gas, we shifted the spectrum to the galaxy rest-frame and used the [O III] $] \lambda \lambda 4958,5007 \AA$ doublet. Each line of the doublet is double peaked and has broad wings (see Fig. 2), clearly requiring multiple components to be modeled. All our fits were performed using Gaussian functions and custom-made IDL routines based on the MPFIT (Markwardt 2009) fitting routine. For each component of the doublet we forced the width of the Gaussians to be the same. In addition, we fixed their separation (49 $\AA$ ) and their relative fluxes $(1: 3)$.

The best-fit model of the [O III] $\lambda \lambda 4958,5007 \AA$ doublet (which is called '[O III] model') was chosen based on $\chi^{2}$ statistics and residual minimization. The [O III] model, shown in Fig. 2 superposed on the observed lines, includes four components:

- A narrow redshifted component $(1 \mathrm{~N})$ with $\mathrm{FWHM}_{1 \mathrm{~N}}=$ $128 \pm 5 \mathrm{~km} \mathrm{~s}^{-1}$ and velocity shift $v_{1 \mathrm{~N}}=99 \pm 35 \mathrm{~km} \mathrm{~s}^{-1}$

- A narrow blueshifted component $(2 \mathrm{~N})$ with $\mathrm{FWHM}_{2 \mathrm{~N}}=$ $104 \pm 4 \mathrm{~km} \mathrm{~s}^{-1}$ and velocity shift $v_{2 \mathrm{~N}}=-80 \pm 35 \mathrm{~km} \mathrm{~s}^{-1}$

- An intermediate component (I) with $\mathrm{FWHM}_{\mathrm{I}}=$ $709 \pm 75 \mathrm{~km} \mathrm{~s}^{-1}$ and velocity shift $v_{\mathrm{I}}=25 \pm 38 \mathrm{~km} \mathrm{~s}^{-1}$

- A very broad blueshifted component $(\mathrm{VB})$ with $\mathrm{FWHM}_{\mathrm{VB}}=$ $2035 \pm 207 \mathrm{~km} \mathrm{~s}^{-1}$ and velocity shift $v_{\mathrm{VB}}=-302 \pm$ $112 \mathrm{~km} \mathrm{~s}^{-1}$

Velocity shifts were calculated with respect to the systemic velocity of the galaxy (i.e., derived from our redshift estimate), and the FWHM of each component is the intrinsic FWHM, taking into account the instrumental spectral resolution.

We derived a model for the permitted hydrogen emission lines using the $\mathrm{H} \beta$ line. Interestingly, the best-fit model for the $\mathrm{H} \beta$ line was consistent with the [O III] model, but required an additional redshifted component with $v=398 \pm 171 \mathrm{~km} \mathrm{~s}^{-1}$ and $\mathrm{FWHM}=1969 \pm 662 \mathrm{~km} \mathrm{~s}^{-1}$. The $\mathrm{H} \beta$ model is shown in Fig. 3 superposed on the observed spectrum. Nevertheless, this additional component was hard to detect in the other hydrogen emission lines in the nuclear spectrum, mainly due to their weakness (e.g., $\operatorname{Pa} \alpha$ ) or because they blend with other emission lines (e.g., $\mathrm{H} \alpha$ and $\mathrm{H} \gamma$ ). A possible explanation is that this component is due to light from the central broad line regions of the AGN scattered by an outflowing dusty medium, which can explain the redshift of the component (see, e.g., di Serego Alighieri et al. 1995; Cimatti et al. 1997; Villar-Martín et al. 2000).

We found that the [O III] model provides a good fit for the $[\mathrm{O}$ II $] \lambda \lambda 3726,29 \AA$, the [O II] $] \lambda \lambda 7319,30 \AA$, the $\left[\mathrm{S}_{\text {II }}\right] \lambda \lambda 4069,76 \AA$ $+\mathrm{H} \delta$, the $\left[\mathrm{S}_{\mathrm{II}}\right] \lambda \lambda 6717,31 \AA$, the $\mathrm{H} \gamma+\left[\mathrm{O}_{\mathrm{III}}\right] \lambda 4363 \AA$, the He ${ }_{\mathrm{II}} \lambda 4686 \AA$, the $\left[\mathrm{N}_{\mathrm{II}}\right] \lambda \lambda 6548,84 \AA+\mathrm{H} \alpha$, the $\left[\mathrm{O}_{\mathrm{I}}\right] \lambda \lambda 6300,63 \AA$,

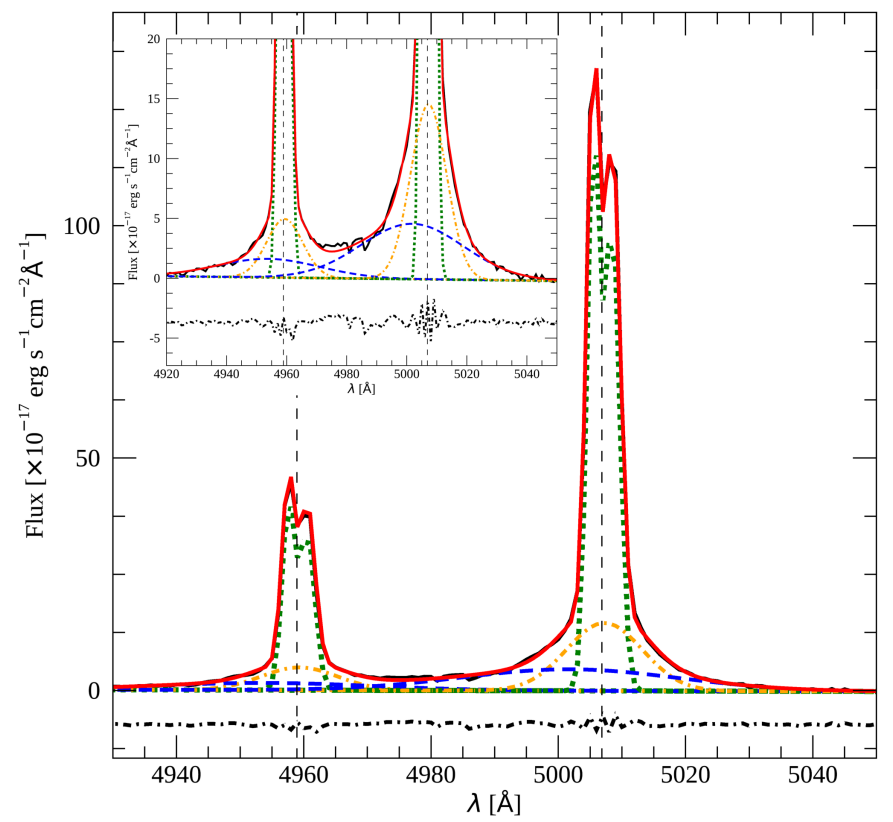

Fig. 2. $[\mathrm{O}$ III] $] \lambda \lambda 4958,5007 \AA$ doublet (black solid line) and the [O III] model (red solid line). The [O III] model includes two narrow components ( $1 \mathrm{~N}$ and $2 \mathrm{~N}$ component, green dotted line), one intermediate component (I component, yellow dot-dashed line), and one very broad component (VB component, blue dashed line). The residuals of the fit are normalized and plotted below the spectrum (black dot-dashed line). The vertical dashed line marks the restframe wavelength of the emission lines. The inset in the top left part of the plot shows a zoom-in of the I and VB components.

the $\left[\mathrm{S}_{\mathrm{III}}\right] \lambda 9531 \AA$ and the Pa $\alpha$ lines. The $\left[\mathrm{O}_{\mathrm{II}}\right] \lambda \lambda 7319,30 \AA$ is a blend of the four [O II] lines at 7319, 7320, 7330, and $7331 \AA$ and was fit as a doublet assuming the line centers to be at $7320.1 \AA$ and 7330.2 $\AA$ (based on Sivjee et al. 1979). The line fluxes of the four components of each emission line are reported in Table A.1.

As has been reported by Holt et al. (2008) and Roche et al. (2016), the line profile of the [O I] $\lambda 6300 \AA$ line shows a redshifted wing and is different from the rest of the forbidden emission line profiles. Using the bright NIR [S III] $\lambda 9531 \AA$, we found that this is due to contamination by the $[\mathrm{S}$ III] $] \lambda 6312 \AA$ line and is not an intrinsic feature of the $\left[\mathrm{O}_{\mathrm{I}}\right] \lambda 6300 \AA$ profile. The [O I $] \lambda 6300 \AA$ line fluxes have been corrected for this when used in line ratio diagnostics (e.g., in Sect. 6). In the case of $\mathrm{H} \gamma$ $+\left[\mathrm{O}_{\text {III }}\right] \lambda 4363 \AA$, we also fit the blend using the $\mathrm{H} \beta$ model instead of the [O III] model for the $\mathrm{H} \gamma$ line. We also note that for He II $\lambda 4686 \AA$ there is no evidence of a very broad component, probably because the line is faint. Additional details on this and on the line fits are reported in Appendix A.

In the NIR part of the nuclear spectrum of PKS B1934-63, we detected the $[\mathrm{Fe}$ II $] \lambda 1.257 \mu \mathrm{m}$ and $\mathrm{Pa} \beta$ lines, which are used in Sect. 6 to study the gas ionization state. The spectrum is noisier and the continuum is not subtracted in the NIR band, which may be the reason that the $\left[\mathrm{O}_{\mathrm{III}}\right]$ model did not give a reliable fit for either of these lines. We fit the $[\mathrm{Fe}$ II $] \lambda 1.257 \mu \mathrm{m}$ line using a single Gaussian function with velocity $v=-95 \pm 49 \mathrm{~km} \mathrm{~s}^{-1}$ and FWHM $=547 \pm 72 \mathrm{~km} \mathrm{~s}^{-1}$. The $\mathrm{Pa} \beta$ line is double peaked, and the best-fitting model included the two narrow components of the [O III] model and a third, broader, component centered at $v=-233 \pm 35 \mathrm{~km} \mathrm{~s}^{-1}$ with an $F W H M=900 \pm$ $193 \mathrm{~km} \mathrm{~s}^{-1}$. 


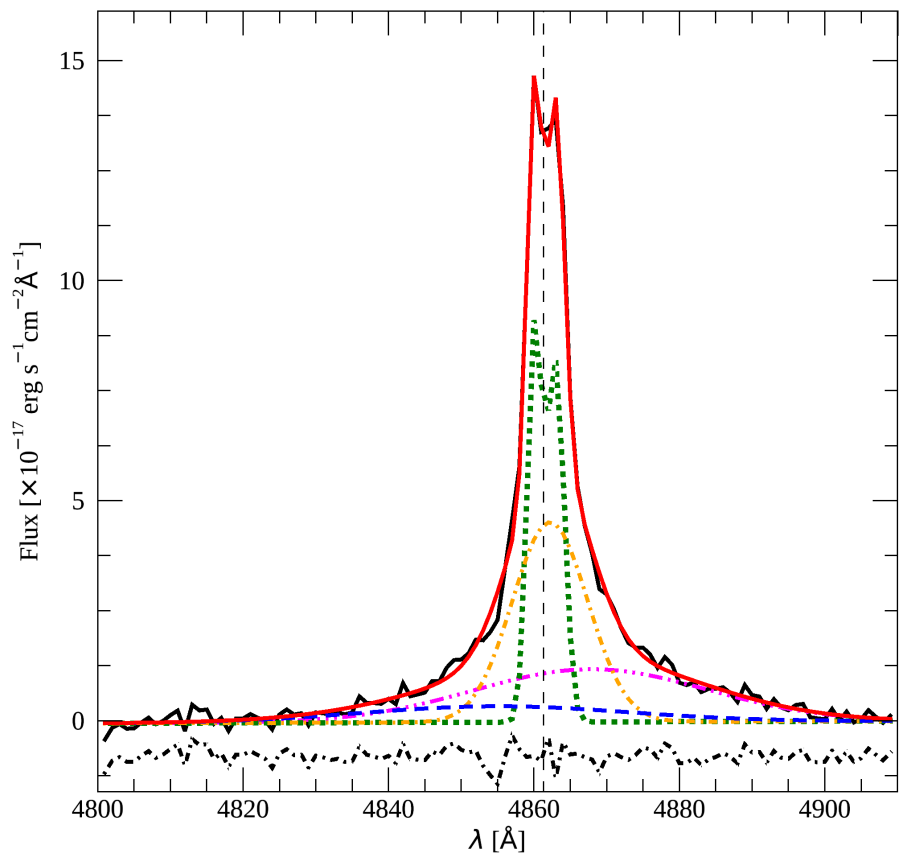

Fig. 3. $\mathrm{H} \beta$ line (black solid line) and the $\mathrm{H} \beta$ model (red solid line). The $\mathrm{H} \beta$ model includes the four components of the [O III] model (see Fig. 2 for a description) and an additional broad redshifted component (magenta triple dot-dashed line). The residuals of the fit are normalized and plotted below the spectrum (black dot-dashed line). The vertical dashed lines marks the restframe wavelength of the emission line.

\subsection{Density diagnostic diagram}

One of our main goals was to derive a robust estimate of the electron density of the warm ionized gas. Our observations have a broad wavelength coverage, which enabled us to use the density diagnostic diagram introduced by Holt et al. (2011). This is based on trans-auroral emission lines, and we used it to estimate the electron density of the four different gas components (see Fig. 4). This diagram uses the [O II] $(3726+3729) /(7319+7330)$ and $[\mathrm{S} \mathrm{II}](4069+4076) /(6717+6731)$ line ratios and also provides an estimate of the gas reddening. As has been discussed in Holt et al. (2011) and Rose et al. (2018), these diagnostics are sensitive to higher densities than the classical line ratios.

Figure 4 shows where the four kinematical components of the warm ionized gas are situated in the diagnostic diagram. To derive values for the gas electron density, we have overplotted AGN photoionization models for different reddening factors. The models were produced using the Cloudy (C13.04, Ferland et al. 2013) photoionization code and the Calzetti et al. (2000) reddening law. The models shown in Fig. 4 have solar metallicity, a photoionizing continuum with $\alpha=-1.5$ and a ionization parameter $U=0.005$, which reproduces typical conditions of an AGN.

Holt et al. (2011) showed that this density diagnostic diagram is not sensitive to the parameters of the AGN photoionization models. This means that the location of the model points in the diagram does not change significantly when the spectral index $\alpha$ of the AGN continuum power law $\left(\mathrm{F}_{v} \propto \gamma^{\alpha}\right)$ and the ionization parameter $U$ are varied.

From the density diagnostic diagram we extracted $\log n_{\mathrm{e}}(1 \mathrm{~N}) \mathrm{cm}^{-3}=2.4 \pm 0.45, \log n_{\mathrm{e}}(2 \mathrm{~N}) \mathrm{cm}^{-3}=2.7 \pm 0.45$ for the two narrow components, $\log n_{\mathrm{e}}(\mathrm{I}) \mathrm{cm}^{-3}=4.6 \pm 0.25$ for the intermediate component, and $\log n_{\mathrm{e}}(\mathrm{VB}) \mathrm{cm}^{-3}=5.5 \pm 0.35$ for the very broad component. The error bars were estimated

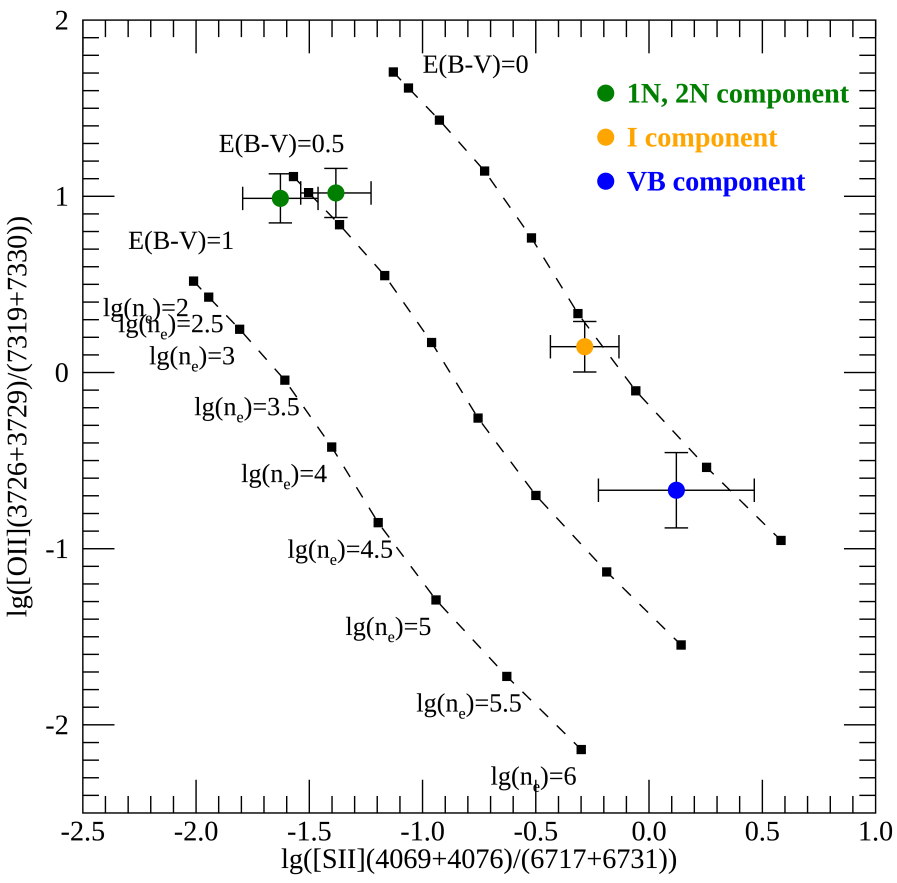

Fig. 4. Density diagnostic diagram using the logarithm of the [O II] $(3727+3729) /(7318+7319+7330+7331)$ and of the [S II] $(4068+4076) /(6716+6731)$ line ratios. Each sequence of black squares, joined by the dashed black line, is a sequence of AGN photoionization models with constant power-law index $(\alpha=-1.5)$ and ionization parameter $(U=0.005)$, created by varying the electron density of the model in the interval $n_{\mathrm{e}}=100-10^{6} \mathrm{~cm}^{-3}$ (from top left to bottom right) with a step $\Delta \log _{10} n_{\mathrm{e}}=0.5$. The three different sequences in the plot (from top right to bottom left) are associated with $E(B-V)=0,0.5$ and 1 . Green circles represent the narrow components; the intermediate and very broad components are indicated by the golden and blue circle, respectively. Error bars for each point are estimated as described in the text.

by summing in quadrature the statistical error from the fitting procedure and the uncertainty in the flux calibration. It is worth mentioning that the $\left[\mathrm{S}_{\mathrm{II}}\right] \lambda 6717 / \lambda 6731$ line ratio, classically used as a density diagnostic, confirmed these results for the narrow components and the intermediate component. The [S $\left.\mathrm{S}_{\text {II }}\right] \lambda 6717 / \lambda 6731$ ratio is $1.10 \pm 0.4$ and $1.03 \pm 0.04$ for the narrow components and decreases to $0.45 \pm 0.04$ for the intermediate component, indicating a density of about $3 \times 10^{2} \mathrm{~cm}^{-3}$ and higher than $10^{4} \mathrm{~cm}^{-3}$, respectively. The signal-to-noise ratio $(\mathrm{S} / \mathrm{N})$ is lower for the very broad component, therefore we could not estimate the density in this way. The density diagnostic diagram shows that the warm ionized gas spans a significant range of densities from $\sim 3 \times 10^{2.0} \mathrm{~cm}^{-3}$ up to $10^{5.5} \mathrm{~cm}^{-3}$, and the higher values are found for the broader components.

The comparison between the observed points and the sequences of models with different $E(B-V)$ values in the density diagnostic diagram allowed us to derive estimates of the reddening of the four kinematical components for the warm ionized gas. We found $E(B-V)_{1 \mathrm{~N}}=0.52 \pm 0.12, E(B-V)_{2 \mathrm{~N}}=0.40 \pm 0.12$ for the narrow components, $E(B-V)_{\mathrm{I}}=0.05 \pm 0.20$ for the intermediate component, and $E(B-V)_{\mathrm{VB}}=0.12 \pm 0.25$ for the very broad component.

We compare these numbers to the reddenings estimated using the classical approach of the hydrogen line ratios (i.e., the Balmer decrement). We used the $\mathrm{H} \alpha / \mathrm{H} \beta$ and the $\mathrm{Pa} \alpha / \mathrm{H} \beta$ line ratios and converted them into a color excess $E(B-V)$ following the approach of Momcheva et al. (2013) and using 
the Calzetti et al. (2000) extinction curve. The errors on the line ratios take into account both the statistical error of the fitting procedure and the uncertainty in the flux calibration. From the $\mathrm{H} \alpha / \mathrm{H} \beta$ line ratio we obtained $E(B-V)_{1 \mathrm{~N}}=0.43 \pm$ $0.135, E(B-V)_{2 \mathrm{~N}}=0.40 \pm 0.135, E(B-V)_{\mathrm{I}}=0.56 \pm 0.137$ and $E(B-V)_{\mathrm{VB}}=0.186 \pm 0.8$. From the $\mathrm{Pa} \alpha / \mathrm{H} \beta$ line ratio we obtained $E(B-V)_{1 \mathrm{~N}}=0.28 \pm 0.11, E(B-V)_{2 \mathrm{~N}}=0.25 \pm 0.11$, $E(B-V)_{\mathrm{I}}=0.11 \pm 0.19$ and $E(B-V)_{\mathrm{VB}}=0.82 \pm 0.85$. Taking into account the uncertainties, these values and the $E(B-V)$ values extracted from trans-auroral lines generally agree well. The large uncertainties of the classical approach are mainly due to the faintness of the $\operatorname{Pa} \alpha$ line (e.g., the difficulty in determining its continuum level) and the complex line blend in which the $\mathrm{H} \alpha$ line is included. We thus preferred to adopt the reddening values coming from the density diagnostic diagram, which are based on the strong emission lines. These values are reported in Table 1 and were used in the estimate of the intrinsic [O III] and $\mathrm{H} \beta$ luminosities of the different gas components.

We found that none of the kinematic components shows high reddening and that the reddening of the intermediate and very broad components is lower than that of the narrow components. This is consistent with the results for some ultraluminous infrared galaxies (ULIRG) in the sample of Rose et al. (2018) but at odds with the results on the compact radio source PKS B1345+12 obtained by Holt et al. (2011), who found higher reddenings for broader components with the same method.

\subsection{Radius of the narrow and broad gas components}

To understand whether the warm ionized gas is extended or concentrated in the central regions of the host galaxy, we used the $\left[\mathrm{O}_{\text {III }}\right] \lambda 5007 \AA$ line and the spatial information contained in the slit spectrum. We extracted spatial profiles for the warm ionized gas components (i.e., one for the two narrow components and one including the intermediate and the very broad components) and compared them to the seeing of our observations.

To extract these profiles, we collapsed the slit spectrum along the spectral direction over a given velocity range (with respect to the systemic velocity). The selected velocity range for the narrow components was $-226 \lesssim v \lesssim 279 \mathrm{~km} \mathrm{~s}^{-1}$. For the intermediate and very broad component we took the velocity range $-854 \lesssim v \lesssim-348 \mathrm{~km} \mathrm{~s}^{-1}$. The profile of the galaxy starlight emission was extracted using two windows, one on the red side $\left(-2031 \lesssim v \lesssim-3044 \mathrm{~km} \mathrm{~s}^{-1}\right)$ and one on the blue side $\left(-5867 \lesssim v \lesssim-4855 \mathrm{~km} \mathrm{~s}^{-1}\right)$ of the [O $\left.\mathrm{III}\right] \lambda \lambda 4958,5007 \AA$ lines.

The host galaxy profile was then corrected for the differences in the widths of the slices and was subtracted from the ionized gas profiles. The residuals were then fitted with a Gaussian function. For both these profiles we obtained an FWHM of $0.95 \pm 0.01$ arcsec, which is consistent with the FWHM of the seeing (i.e., $0.97 \pm 0.06$ arcsec, see Sect. 2). This indicates that the warm ionized gas (of all the kinematical components) is not spatially resolved by our observations using this technique and is concentrated in the nuclear regions of the host galaxy.

To obtain an upper limit on the radius of the warm ionized gas, we used the following equation:

$r \leq \frac{1}{2} \sqrt{(\mathrm{FWHM}+3 \sigma)^{2}-\mathrm{FWHM}^{2}}$,

where the FWHM is the seeing and $\sigma$ is the uncertainty on the seeing. We obtained $r \leq 0.3$ arcsec, which at the redshift of the galaxy is equivalent to $r \leq 955 \mathrm{pc}$.

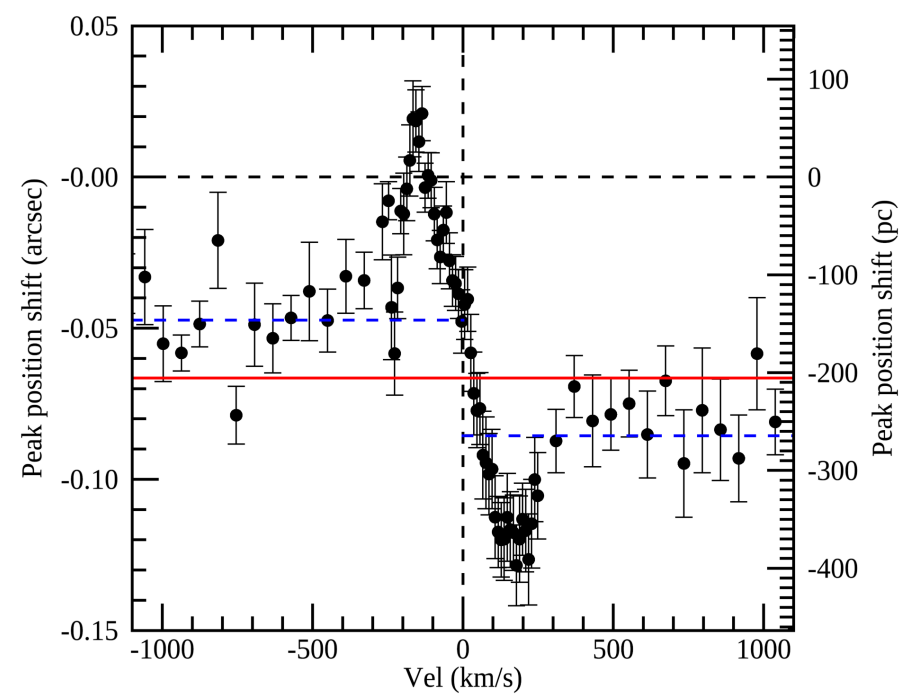

Fig. 5. Fitted centers of the [O III] $5007 \AA$ emission line spatial profiles, measured in arcsec (left $y$-axis) and parsec (right $y$-axis), as a function of velocity. The black dashed vertical and horizontal lines mark the zero point of both axes. The blue dashed lines mark the errorweighted mean position of the [O III] spatial profiles at $v<250 \mathrm{~km} \mathrm{~s}^{-1}$ and at $v>250 \mathrm{~km} \mathrm{~s}^{-1}$, and the red solid line marks their average. The negative values along the $y$-axis indicate the direction pointing toward the companion galaxy to the west.

\section{Gas kinematics in the inner regions}

Using the spectro-astrometry technique, we studied the spatial extents of the different ionized gas components, overcoming the limitations given by the seeing. Spectro-astrometry uses high $\mathrm{S} / \mathrm{N}$ long-slit spectra to measure the centroid position of an unresolved object as function of wavelength. It is based on the fact that the centroid position can be measured with much higher precision than the seeing-limited spatial resolution of the observations (Bailey 1998b), and has been used to identify and study binary stars (see Bailey 1998a; Takami et al. 2003).

We used this technique on the high S/N [O III $] \lambda 5007 \AA$ line in our slit spectrum and investigated how the warm ionized gas at different velocities is distributed along the slit in the spatial direction. In this way, we probed the gas distribution at subarcsecond scales, which at the redshift of PKS B1934-63 correspond to scales of tens of parsec. In the slit spectrum, we isolated the region around the $[\mathrm{O}$ III $] \lambda 5007 \AA$ line, and for a given pixel along the spectral direction, we extracted a profile of the ionized gas along the spatial direction. Every spatial profile probes warm ionized gas at a different velocity. Then, we fit each extracted spatial profile with a Gaussian function and used the fitted profile center to establish the spatial location of the gas at that specific velocity.

We used an average spatial profile of the galaxy starlight to locate the host galaxy center, and we took this as a reference point to establish the location of the ionized gas. We also subtracted the starlight spatial profile from the ionized gas spatial profiles to avoid contamination from the light of the host galaxy. This was particularly important for the profiles extracted at velocities $v<-250 \mathrm{~km} \mathrm{~s}^{-1}$ and $v>250 \mathrm{~km} \mathrm{~s}^{-1}$, where the fainter $\left[\mathrm{O}_{\text {III }}\right] \lambda 5007 \AA$ emission of the broad (i.e., the intermediate and very broad) components of the warm ionized gas is located. To increase the $\mathrm{S} / \mathrm{N}$ of the gas spatial profiles at these velocities, we binned the data along the spectral direction using a box that was three pixels wide. For gas at velocities $-250<v<250 \mathrm{~km} \mathrm{~s}^{-1}$, we instead extracted a spatial profile for every pixel along the spectral direction. 
F. Santoro et al.: Probing multi-phase outflows and AGN feedback in compact radio galaxies: the case of PKS B1934-63

Table 1. Kinematical and physical properties of the four kinematical components found for the warm ionized gas.

\begin{tabular}{lllll}
\hline \hline & 1N Component & 2N Component & I Component & VB Component \\
\hline$v\left[\mathrm{~km} \mathrm{~s}^{-1}\right]$ & $99.6 \pm 35.4$ & $-80 \pm 35.4$ & $25 \pm 38.5$ & $-302 \pm 112$ \\
FWHM $\left[\mathrm{km} \mathrm{s}^{-1}\right]$ & $128 \pm 5.3$ & $104 \pm 4.2$ & $709 \pm 75.3$ & $2035 \pm 207$ \\
$\log n_{\mathrm{e}}\left[\mathrm{cm}^{-3}\right]$ & $2.4 \pm 0.45$ & $2.7 \pm 0.45$ & $4.6 \pm 0.25$ & $5.5 \pm 0.35$ \\
$E(B-V)$ & $0.52 \pm 0.125$ & $0.4 \pm 0.125$ & $0.05 \pm 0.2$ & $0.125 \pm 0.25$ \\
$L(\mathrm{H} \beta)\left[\mathrm{erg} \mathrm{s}^{-1}\right]$ & $(1.89 \pm 0.20) \times 10^{41}$ & $(1.25 \pm 0.12) \times 10^{41}$ & $(6.93 \pm 0.70) \times 10^{40}$ & $(2.67 \pm 0.26) \times 10^{40}$ \\
$M_{\text {gas }}\left[M_{\odot}\right]$ & $(5.1 \pm 0.5) \times 10^{6}$ & $(1.7 \pm 0.2) \times 10^{6}$ & $(1.2 \pm 0.1) \times 10^{4}$ & $(5.7 \pm 0.5) \times 10^{2}$ \\
\hline
\end{tabular}

Notes. The central velocity $v$ and FWHM are obtained from the [O III] model, the electron density $n_{\mathrm{e}}$ and reddening $E(B-V)$ values are extracted using the density diagnostic diagram in Sect. 3.3, $L(H \beta)$ is the reddening corrected $\mathrm{H} \beta$ luminosity, and $M_{\text {gas }}$ is the mass of warm ionized gas estimated in Sect. 5.

In Fig. 5 we show the spatial position of the fitted centers of the gas spatial profiles (expressed in arcsec/pc) as a function of the velocity associated with each profile. The zero point along the $\mathrm{x}$-axis is the systemic velocity of the galaxy, while the zero point along the $y$-axis is the fitted center of the galaxy spatial profile.

The S-shaped trend we see in Fig. 5 for the two narrow components (i.e., at $-250<v<250 \mathrm{~km} \mathrm{~s}^{-1}$ ) might be explained by a disk-like structure or a biconical outflow in the central regions of the galaxy (up to about $\pm 200 \mathrm{pc}$ ). The curve is symmetric around the zero velocity value and reaches a maximum spatial shift at about $\pm 150 \mathrm{~km} \mathrm{~s}^{-1}$. The overall curve is clearly spatially shifted with respect to the zero point along the $y$-axis (i.e., the center of the galaxy), and this might be explained by the effect of obscuring dust, which can potentially shift the position of the galaxy spatial profile peak that we used as an indicator of the true AGN nucleus position.

Unlike Roche et al. (2016), we do not find evidence for gas emission on large scales $(>1 \mathrm{kpc})$. However, if the inner structure we detect from spectro-astrometry is a circum-nuclear disk (CND), it would rotate in the same sense as the more extended gas disk seen by Roche et al. (2016), with the western gas rotating away from the observer. Our slit is not aligned with the major axis of the structure that Roche et al. (2016) observed, which may explain why this is, instead, spatially unresolved by our observations, as shown in Sect. 3.4. The CND of warm and cold gas, extended on scales of a few hundreds of parsec, at the center of AGN has previously been found in studies of, for example, Dumas et al. (2007); Hicks et al. (2013); Maccagni et al. (2016) and García-Burillo et al. (2016), and it has been proposed that these structures constitute the reservoir of gas from which the $\mathrm{BH}$ feeds. It is worth noting that the amplitude of the rotation that we find is larger than reported in Roche et al. (2016). The reason is probably that their observations do not resolve the double peak of the $\left[\mathrm{O}_{\mathrm{III}}\right] \lambda 5007 \AA$ line and they used a single Gaussian fit to derive the [O $\mathrm{III}] \lambda 5007 \AA$ velocity field, which smoothes out the velocity gradient that we observe. Considering this, it is plausible that the CND we observe, and the largescale disk found by Roche et al. (2016), are part of the same disk-like structure whose kinematical axis is misaligned with respect to the axis of the radio source (see Fig. 31 in Roche et al. 2016.

On the other hand, the gas at $v<-250 \mathrm{~km} \mathrm{~s}^{-1}$ and at $v>250 \mathrm{~km} \mathrm{~s}^{-1}$ is associated with the intermediate and the very broad components and is representative of the warm ionized gas that is outflowing. The error-weighted mean positions of the gas at $v<-250 \mathrm{~km} \mathrm{~s}^{-1}$ and at $v>250 \mathrm{~km} \mathrm{~s}^{-1}$ are $-0.047 \pm 0.002$ and $-0.085 \pm 0.003$, respectively (see Fig. 5). This spatial asymmetry supports the idea that the geometry of the outflowing gas is biconical. Assuming a biconical geometry (i.e., the blueshifted and redshifted gas emission comes from the two sides of the nucleus), we can estimate the position of the nucleus (i.e., the red horizontal line in Fig. 5) and form an idea of the radius of the outflowing gas. In this way, we find that the outflow has a radius of $59 \pm 12 \mathrm{pc}$. This matches the radial extent of the radio source well (the separation between the radio lobes of PKS B1934-63 is $131,7 \pm 0.9 \mathrm{pc}$ ) and suggests that the warm ionized gas is outflowing as a consequence of the interaction with the radio jets. Given the orientation of the radio source, it is likely that the jets are accelerating part of the gas in regular rotation in the CND. Together with the findings of Roche et al. (2016), this suggests that the velocity gradient of the kinematically disturbed gas is aligned along the radio axis.

We are aware that taking only the average position for the gas in the broad wings of the $\left[\mathrm{O}_{\mathrm{III}}\right] \lambda 5007 \AA$ line might underestimate the size of the outflow. However, even considering the higher absolute value for the shift of the gas at $v<-200 \mathrm{~km} \mathrm{~s}^{-1}$ and at $v>200 \mathrm{~km} \mathrm{~s}^{-1}$ with respect to the estimated position of the nucleus, we find that if we were to include the errors on the spatial shifts, the outflow would have a maximum radius of $\sim 175 \mathrm{pc}$. This indicates that the outflow is extended on spatial scales that are comparable with the size of the radio source.

We can exclude that the motions of the gas in the broad wings of the $\left[\mathrm{O}_{\text {III }}\right] \lambda 5007 \AA$ line are due to rotation around the central $\mathrm{BH}$. If the gas at velocities between 500 and $1000 \mathrm{~km} \mathrm{~s}^{-1}$ is located at a distance of about $60 \mathrm{pc}$ from the central $\mathrm{BH}$, a BH mass in the interval $0.34-1.35 \times 10^{10} M_{\odot}$ would be required. Such masses are too high for the $\mathrm{BH}$ of a galaxy with a stellar mass of about $10^{11} M_{\odot}$ (as estimated by Roche et al. 2016), which according to scaling relations (Kormendy \& Ho 2013), is expected to host a BH with a mass of about $5 \times 10^{8} M_{\odot}$.

As a sanity check, we also performed a spectro-astrometry analysis on the $\left[\mathrm{O}_{\text {III }}\right] \lambda 4958 \AA$ line. The gas at $-250<v<$ $250 \mathrm{~km} \mathrm{~s}^{-1}$ confirms the trend seen for the [O III $] \lambda 5007 \AA$ line. However, although the lower $\mathrm{S} / \mathrm{N}$ of the broad wings of the line does not allow us to confirm the exact spatial extent of the outflow component, in line with our findings for the [O III] $\lambda 5007 \AA$ line, we can see that this gas is extended on smaller spatial scales than the gas at low velocities.

\section{Warm ionized gas and outflow parameters}

We estimated the warm ionized gas mass of the different kinematical components using the following equation:

$M_{\mathrm{gas}}=\frac{L(\mathrm{H} \beta) m_{\mathrm{p}}}{n_{\mathrm{e}} \alpha_{\mathrm{H} \beta}^{\mathrm{eff}} h v_{\mathrm{H} \beta}}$, 
where $L(\mathrm{H} \beta)$ is the $\mathrm{H} \beta$ luminosity corrected for dust extinction, $m_{\mathrm{p}}$ is the proton mass, $n_{\mathrm{e}}$ is the electron density from the density diagnostic diagram, $\alpha_{\mathrm{H} \beta}^{\text {eff }}$ is the effective $\mathrm{H} \beta$ recombination coefficient (taken as $3.03 \times 10^{-14} \mathrm{~cm}^{3} \mathrm{~s}^{-1}$ for case B in the low-density limit; Osterbrock \& Ferland 2006), $v_{\mathrm{H} \beta}$ is the frequency of the $\mathrm{H} \beta$, and $h$ is the Planck constant. The $L(\mathrm{H} \beta)$ and the estimated $M_{\text {gas }}$ of each kinematical component are reported in Table 1.

We found that the two narrow components have a mass of warm ionized gas of $M_{\text {gas }}(1 \mathrm{~N})=(5.1 \pm 0.5) \times 10^{6} M_{\odot}$ and $M_{\text {gas }}(2 \mathrm{~N})=(1.7 \pm 0.2) \times 10^{6} \quad M_{\odot}$. The intermediate component has a gas mass of $M_{\text {gas }}(\mathrm{I})=(1.2 \pm 0.1) \times 10^{4} M_{\odot}$, while for the very broad component, we found $M_{\text {gas }}(\mathrm{VB})=(5.7 \pm$ $0.5) \times 10^{2} M_{\odot}$. It is clear that almost the entire reservoir of the host galaxy's warm ionized gas is found in the two narrow components; the intermediate and the very broad components represent only a small fraction of the warm ionized gas reservoir.

With a reliable estimate of the electron density of the outflowing warm ionized gas, we were able to characterize the properties of the outflow. Following the method described in Sect. 4.1 of Rose et al. (2018), we determined the mass outflow rate $\dot{M}$, the outflow kinetic power $\dot{E}$ and the AGN feedback efficiency $F_{\text {kin }}=\dot{E} / L_{\text {bol }}$ using the following formulae:

$\dot{M}=\frac{L(\mathrm{H} \beta) m_{\mathrm{p}} v_{\text {out }}}{n_{\mathrm{e}} \alpha_{\mathrm{H} \beta}^{\mathrm{eff}} h v_{\mathrm{H} \beta} r}$

$\dot{E}=\frac{\dot{M}}{2}\left(v_{\text {out }}^{2}+3 \sigma^{2}\right)$

where $v_{\text {out }}$ is the velocity, $r$ is the radius, and $\sigma$ is the line-of-sight velocity dispersion $(\sigma=F W H M / 2.355)$ of the outflow.

To estimate these parameters, we used the radii estimated in Sect. 3.4 (i.e. $r<955 \mathrm{pc}$ ) and in Sect. 4 (i.e., $r>60 \mathrm{pc}$ ) as upper and lower limits for the radius of the outflow, respectively. We extracted the bolometric luminosity using the dereddened [O III $] \lambda 5007 \AA$ total luminosity, which is usually considered a good indicator of the AGN power (see Heckman et al. 2004). Summing the intrinsic [O III] $\lambda 5007 \AA$ luminosities of each component, we obtained $\left.L_{[\mathrm{O}} \mathrm{II}\right]=(4.4 \pm 0.4) \times 10^{42} \mathrm{erg} \mathrm{s}^{-1}$. To extract the bolometric luminosity, we used the bolometric correction of Lamastra et al. (2009), $L_{\mathrm{bol}}=454 L_{\left[\mathrm{O}_{\mathrm{III}}\right]}$, which is valid for object with intrinsic [O III] $\lambda 5007 \AA$ luminosity in the interval $L_{[\mathrm{O} \text { II] }]}=10^{42-44} \mathrm{erg} \mathrm{s}^{-1}$. This resulted in a bolometric luminosity of $L_{\mathrm{bol}}=(2 \pm 0.2) \times 10^{45} \mathrm{erg} \mathrm{s}^{-1}$, which makes PKS B1934-63 a type II quasar according to the criterion of Zakamska et al. (2003).

We estimated the outflow properties for the gas emitting the very broad component and for both of the broader components together (i.e., the intermediate and very broad component). In the latter case, for $L(H \beta)$ we took the intrinsic integrated $H \beta$ luminosity of the two components, while for $n_{\mathrm{e}}, v_{\text {out }}$, and the FWHM of the gas, we took a flux-weighted value. All the relevant quantities for these calculations are reported in Table 2 together with the estimated $\dot{M}, \dot{E}$, and $F_{\text {kin }}$. In this way, we obtained mass outflow rates in the range $10^{-3}-10^{-4} M_{\odot} \mathrm{yr}^{-1}$ and AGN feedback efficiencies in the range $10^{-4}-10^{-5} \%$.

This approach assumed that the central velocity of the line associated with the outflowing gas is the true velocity of the outflow $v_{\text {out }}$ and its broadening is due to the gas turbulence. A less conservative approach, which can potentially take into account projection effects, considers that the broadening of the lines is due to the different projections of the velocity vectors of the gas in a quasi-spherical outflow. In this case, the actual outflow velocity $v_{\text {out }}$ is the maximum velocity that the gas reaches in the wings of the emission line profile. To estimate the maximum velocity of the gas, we followed the approach of Rose et al. (2018) and took the velocity corresponding to a $5 \%$ cut of the flux in the blueshifted direction. We report all the relevant quantities for the calculations for this case as well, together with the estimated $\dot{M}, \dot{E}$, and $F_{\text {kin }}$ in Table 2. With this method we obtained mass outflow rates and AGN feedback efficiencies for the intermediate and very broad components, which are, on average, one order of magnitude higher than the results obtained with the first approach.

Both methods gave AGN feedback efficiencies that are among the lowest found for warm ionized gas outflows (see Fig. 2 in Harrison et al. 2018). Our values are far from the 5-10\% required by the classical AGN feedback models (e.g., Fabian 1999; Di Matteo et al. 2005; Springel et al. 2005) and also lower that the $0.5 \%$ of the multi-staged model proposed by Hopkins \& Elvis (2010). However, as stressed by Harrison et al. (2018), there are some caveats to consider when comparing the AGN feedback efficiency derived from observations to the prescription of cosmological models, especially because the energy that is actually transferred to the warm and cool ISM can be a fraction of the energy injected by the AGN into the surrounding medium.

\section{Gas ionization}

Given that the gas in the intermediate and the very broad components shows remarkable differences in terms of densities and kinematics from the rest of the warm ionized gas, we investigated whether these components also stand out in terms of their ionization properties.

In Figs. 6 and 7 we present the classical BPT diagrams originally introduced by Baldwin et al. (1981) with the observed line ratios of the different gas kinematical components. As expected, the observed line ratios of the four kinematical components are typical of AGN, although with some differences between the components. As in the case of the density diagnostic diagram, the two narrow components have similar line ratios (see Fig. 6), confirming that they are part of the same structure.

All the gas components, but in particular the very broad component, have high values of the $\left[\mathrm{O}_{\mathrm{I}}\right] \lambda 6300 / \mathrm{H} \alpha$ line ratio, higher than those usually measured in $\mathrm{AGN}$, and they are indicative of shock-ionized gas. The [O I] $\lambda 6300 \AA$ line is emitted by warm weakly ionized gas that is typically located in the transition region between ionized gas and neutral gas. Only high-energy photons can penetrate this region, which stimulates the $\left[\mathrm{O}_{\mathrm{I}}\right] \lambda 6300 \AA$ emission, and a source of such high-energy photons can be the AGN continuum radiation, shocks, or a combination of the two.

We investigated the gas ionization mechanisms by comparing the observed line ratios with model grids of AGN photoionization and of shocks. The model grids were taken from the ITERA tool (Groves \& Allen 2010) and created using MAPPINGS III (Sutherland et al. 2013). The AGN photoionization model grids (see Fig. 6 and the upper panel in Fig. 7) were obtained by varying the spectral index $\alpha$ from -2 to -1.2 and the ionization parameter $\log U$ from -4 to 0 . The shock models (see the lower panel in Fig. 7) included in the ITERA tool were taken from Allen et al. (2008), they have solar metallicity and cover shock velocities in the range $v_{\mathrm{s}}=100-1000 \mathrm{~km} \mathrm{~s}^{-1}$ and magnetic fields (i.e., pre-shock transverse magnetic field) in the range $B=0.01-1000 \mu \mathrm{G}$.

In Fig. 6 we compare the line ratios of the narrow components to AGN photoionization models with gas density $n_{\mathrm{e}}=10^{3} \mathrm{~cm}^{-3}$ (according to the densities derived in Sect. 3.3) and both solar 
Table 2. Mass outflow rates $\dot{M}$, outflow kinetic energy $\dot{E}$, and AGN feedback efficiency $F_{\text {kin }}=\dot{E} / L_{\mathrm{bol}}$ obtained with the two different methods described in the text, together with all the relevant quantities used in the calculation.

\begin{tabular}{|c|c|c|c|c|}
\hline & VB Component & I+VB Component & $\begin{array}{l}\text { VB Component } \\
\mathrm{V}_{\max }\end{array}$ & $\begin{array}{c}\text { I Component } \\
\mathrm{v}_{\max }\end{array}$ \\
\hline$L(\mathrm{H} \beta)\left[\operatorname{erg~s}^{-1}\right]$ & $2.6 \times 10^{40}$ & $9.6 \times 10^{40}$ & $2.6 \times 10^{40}$ & $6.9 \times 10^{40}$ \\
\hline$v_{\text {out }}\left[\mathrm{km} \mathrm{s}^{-1}\right]$ & 302 & 156 & 2462 & 777 \\
\hline$n_{\mathrm{e}}\left[\mathrm{cm}^{-3}\right]$ & $1 \times 10^{5.5}$ & $1.7 \times 10^{5}$ & $1 \times 10^{5.5}$ & $1 \times 10^{4.6}$ \\
\hline FWHM $\left[\mathrm{km} \mathrm{s}^{-1}\right]$ & 2035 & 568 & - & - \\
\hline$\dot{M}\left[M_{\odot} \mathrm{yr}^{-1}\right]$ & $0.002-0.0002$ & 0.009-0.0006 & $0.0015-0.02$ & $0.01-0.14$ \\
\hline$\dot{E}\left[\operatorname{erg~s}^{-1}\right]$ & $(0.13-2.0) \times 10^{39}$ & $(0.2-6.7) \times 10^{39}$ & $(0.29-4.2) \times 10^{40}$ & $(0.18-2.7) \times 10^{40}$ \\
\hline$F_{\text {kin }}$ & $(0.07-1) \times 10^{-6}$ & $(0.1-3.3) \times 10^{-6}$ & $(0.14-2.11) \times 10^{-5}$ & $(0.1-1.3) \times 10^{-5}$ \\
\hline
\end{tabular}

Notes. Columns 2 and 3 report the values obtained using the central velocity of the outflowing gas as $v_{\text {out }}$, while Cols. 4 and 5 report the values obtained using the maximum velocity $v_{\max }$ as $v_{\text {out }}$.

and twice solar (i.e., $2 Z_{\odot}$ ) metallicity. The comparison of the line ratios with the photoionization models provides good evidence for super-solar metallicities in the near-nuclear regions. We also found a good consistency between the positions of the points and the models (in $U$ and $\alpha$ ) in all three plots for twice solar metallicity. The narrow components do not show kinematical evidence of shocked gas (i.e., broad line width), and for this reason, we did not compare their line ratios with shock models.

It is possible that the gas of the narrow components is part of the shock precursor. However, for solar metallicities, precursor models have line ratios similar to the AGN photoionization models and would also fail to explain the observed line ratios. We cannot comment on precursor models with metallicities higher than solar because there are no such models.

In Fig. 7 we use both AGN photoionization and shock models to study the ionization state of the gas in the intermediate and the very broad components. We considered AGN photoionzation models with a gas electron density $n_{\mathrm{e}}=10^{5} \mathrm{~cm}^{-3}$, this model grid was not included in the ITERA tool and was produced using Cloudy (C13.04, Ferland et al. 2013) photoionization code. For the shock models we assumed a shock compression factor of 100 and took a pre-shock gas electron density of $n_{\mathrm{e}}=10^{3} \mathrm{~cm}^{-3}$. The assumed compression factor was intended to take into account the fact that while shock conditions cause a modest density jump (maximum factor $\sim 5$, see Fig. 7 in Sutherland \& Dopita 2017), the temperature jump will further increase the pressure and thus the compression of the gas in the post-shock regions. We found that overall, the shock models reproduce the observed line ratios in the three BPT diagrams (see Fig. 7) better than the AGN photoionization models. In particular, the $\left[\mathrm{S}_{\mathrm{II}}\right] \lambda \lambda 6717,31 / \mathrm{H} \alpha$ and the $\left[\mathrm{O}_{\mathrm{I}}\right] \lambda 6300 / \mathrm{H} \alpha$ line ratios of the intermediate component can be explained by shock models with velocities $v_{\mathrm{s}}=400-500 \mathrm{~km} \mathrm{~s}^{-1}$.

The line ratios of the very broad component were more difficult to explain with current models. The outflowing gas of the very broad component stands out from the rest of the warm ionized gas in terms of both the $\left[\mathrm{N}_{\mathrm{II}}\right] \lambda 6584 / \mathrm{H} \alpha$ and the $\left[\mathrm{O}_{\mathrm{I}}\right] \lambda 6300 / \mathrm{H} \alpha$ line ratios, but not in terms of the $[\mathrm{S}$ II] $] \lambda 26717,31 / \mathrm{H} \alpha$ ratio. This can be explained with the high densities that are associated with this gas component and with the fact that the [ $\mathrm{S}_{\mathrm{II}}$ ] lines have a lower critical density (about $5 \times 10^{3} \mathrm{~cm}^{-3}$, Zheng 1988) than the [N II] and [OI] emission lines. Considering the trend of the model grids, the $\left[\mathrm{O}_{\mathrm{I}}\right] \lambda 6300 / \mathrm{H} \alpha$ line ratio is consistent with shocks of higher velocities $\left(v_{s} \geq 1000 \mathrm{~km} \mathrm{~s}^{-1}\right)$, as is also suggested by the extreme kinematics of the gas of the very broad component. On the other hand, the high value of the $\left[\mathrm{N}_{\mathrm{II}}\right] \lambda 6584 / \mathrm{H} \alpha$ line ratio might be an indication of and enhanced N/O ratio (i.e., higher than solar) for the gas of the very broad component (see, e.g., Tadhunter et al. 1994a; Matsuoka et al. 2017).

We are aware that the variation in the shock compression factor and gas metallicity plays a role in the final line ratios of the models. In addition, degeneracies in the fitting of the intermediate and very broad components in the $\left[\mathrm{N}_{\mathrm{II}}\right]+\mathrm{H} \alpha$ blend may contribute to the extreme $\left[\mathrm{O}_{\mathrm{I}}\right] / \mathrm{H} \alpha$ and $\left[\mathrm{N}_{\mathrm{II}}\right] / \mathrm{H} \alpha$ ratios that are observed. New shock models with a fully self-consistent treatment of the pre-shock ionization and thermal structure are being developed for fast shocks and high gas electron densities (Sutherland \& Dopita 2017).

The $[\mathrm{O}$ III] $] \lambda \lambda 4958,5007 / 4363$ ratio, classically used as a temperature diagnostic, together with the $\mathrm{He} \operatorname{II} \lambda 4686 / \mathrm{H} \beta$ ratio give us further indications that the temperature of the gas of the intermediate component is high and that this is due to shocks. Unfortunately, we cannot comment on the temperatures associated with the gas of the very broad component because the detection of this component in the [O III] $\lambda 4363 \AA$ line depends on the model used to fit the $\mathrm{H} \gamma$ line. When we add a broad redshifted component for $\mathrm{H} \gamma$ (according to what we find for the $\mathrm{H} \beta$ line) to the fitting procedure, the very broad component of the $\left[\mathrm{O}_{\text {III }}\right] \lambda 4363 \AA$ line is not detected (see Fig. A.11). In addition, the very broad component is not detected in the case of the He II $\lambda 4686 \AA$ emission line (see Fig. A.12).

By taking into account the main error sources in the fluxes of the $\left[\mathrm{O}_{\mathrm{III}}\right] \lambda 4363 \AA$ components (i.e., the model used for the fitting of the $\mathrm{H} \gamma$ line and the level of the line continuum), we obtain an $\left[\mathrm{O}_{\text {III }}\right] \lambda \lambda 4958,5007 / 4363$ ratio of $70 \pm 2$ and $104 \pm 3$ for the two narrow components and of $30 \pm 3.6$ for the intermediate component. To derive an electron temperature for the gas of the narrow components and the gas of the intermediate component, we used the formula given in Osterbrock \& Ferland (2006) and an electron density of $n_{\mathrm{e}}=10^{3} \mathrm{~cm}^{-3}$ and $n_{\mathrm{e}}=10^{4.5} \mathrm{~cm}^{-3}$, respectively, according to our findings in Sect. 3.3. We find that these line ratios correspond to gas electron temperatures of $T_{\mathrm{e}}(1 N)=15100 \pm 200 \mathrm{~K}, T_{\mathrm{e}}(2 N)=13000 \pm 200 \mathrm{~K}$, and $T_{\mathrm{e}}(I)=30175 \pm 4400 \mathrm{~K}$.

As shown by the models of Binette et al. (1996), it is possible to have high electron temperatures in the presence of matter-bounded clouds that are photoionized by the AGN continuum. However, as stressed in Villar-Martín et al. (1999), these models cannot explain temperatures higher than 

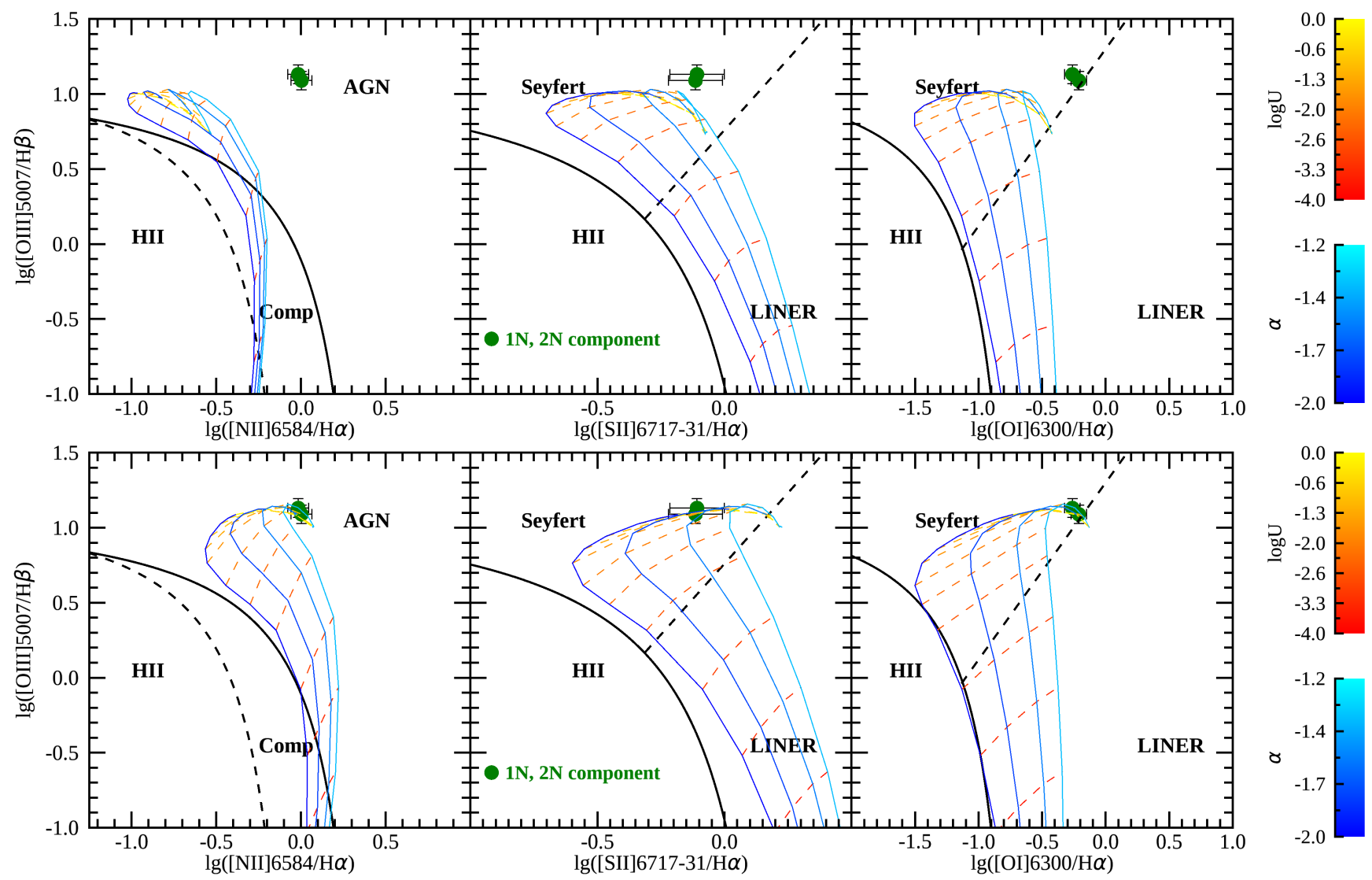

Fig. 6. BPT diagrams for the two narrow components with models of AGN photoionization with solar metallicity (upper panels) and twice the solar metallicity (lower panels). The models have a gas density $n_{\mathrm{e}}=10^{3} \mathrm{~cm}^{-3}$, the dashed lines indicate models with constant photoionization parameter $\log U$ (from -4 to 0 , from bottom to top ), and the solid lines refer to models with constant spectral index $\alpha$ (from -2 to -1.2 , from left to right). The solid line in all the panels is the Kewley et al. (2001) maximum starburst line. The dashed line in the left panels is the semi-empirical Kauffmann et al. (2003) line. The dashed line in the central and right panels is the empirical Kewley et al. (2006) line separating Seyfert galaxies from LINERS.

$20000 \mathrm{~K}$ and a $\mathrm{He} \mathrm{II} \lambda 4686 / \mathrm{H} \beta$ ratios lower than about $0.3-$ 0.4 simultaneously. For the intermediate component we find a He II $\lambda 4686 / \mathrm{H} \beta=0.07 \pm 0.04$, which together with the high temperature favors shocks as the mechanism that heats the gas, in agreement with our findings from the BPT diagram shown in Fig. 7.

Additional evidence for shocks is also provided by the $[\mathrm{Fe}$ II $] \lambda 1.257 \mu \mathrm{m} / \mathrm{Pa} \beta$ line ratio. This line ratio can indicate whether shock ionization produces the $\left[\mathrm{Fe}_{\mathrm{II}}\right]$ emission. This was first suggested by Forbes \& Ward (1993) and Blietz et al. (1994), who found a correlation between the [Fe II] and the radio emission in radio AGN. It has been shown that in galaxies hosting an $\mathrm{AGN}$, a $\left[\mathrm{Fe} \mathrm{II}_{\mathrm{II}}\right] \lambda 1.257 \mu \mathrm{m} / \mathrm{Pa} \beta$ ratio close to 0.6 is produced by AGN photoionization, while a ratio close to 2 is related to shock excitation (Storchi-Bergmann et al. 1999; Rodríguez-Ardila et al. 2004).

In the NIR band of the nuclear spectrum of PKS B1934-63, we detected both the $[\mathrm{Fe}$ II $] \lambda 1.257 \mu \mathrm{m}$ and $\mathrm{Pa} \beta$ lines. Because their $\mathrm{S} / \mathrm{N}$ is low, we were unable to fit the lines with the [O III] model (see Fig. A.13), and we extracted a line ratio using the total flux of the two lines. We find $[\mathrm{Fe}$ II $] 1.257 / \mathrm{Pa} \beta=1.44 \pm 0.2$, which is indicative of shock-ionized gas.

Overall, the combination of the diagnostic diagrams presented in Fig. 7 and the $\left[\mathrm{O}_{\text {III }}\right] \lambda \lambda 4958,5007 / 4363$, He ${ }_{\mathrm{II}} \lambda 4686 / \mathrm{H} \beta$ and $[\mathrm{Fe}$ II] $\lambda 1.257 \mu \mathrm{m} / \mathrm{Pa} \beta$ ratios strongly support the idea that the intermediate outflow component is shockionized.

\section{7. $\mathrm{H}_{2}$ warm molecular and the neutral gas}

Compact steep-spectrum radio sources are known to host massive outflows of molecular and atomic gas (see, e.g., Morganti et al. 2005a; Dasyra \& Combes 2012; Tadhunter et al. 2014). Probing only the warm ionized gas phase, we might be missing part of the gas that is outflowing. The broad wavelength range covered by $\mathrm{X}$-shooter enabled us to probe the kinematics of the warm molecular gas via the $\mathrm{H}_{2}$ emission lines detected in the NIR band. In the nuclear spectrum of PKS B1934-63, we identified the $\mathrm{H}_{2} \mathrm{~S}(5) 1-0$ line at $1.835 \mu \mathrm{m}$, the $\mathrm{H}_{2} \mathrm{~S}(4) 1-0$ line at $1.891 \mu \mathrm{m}$, and the $\mathrm{H}_{2} \mathrm{~S}(3) 1-0$ line at $1.957 \mu \mathrm{m}$. Figure 8 clearly shows that the $\mathrm{H}_{2}$ emission lines are narrower than the warm ionized gas emission lines (e.g., the $\mathrm{Pa} \alpha$ line).

To provide an overview of the kinematics of the different phases of the gas, in Fig. 9 we compare the kinematics of the warm ionized gas with the kinematics of the warm molecular and atomic gas by plotting the normalized line profiles of the [O III] $\lambda 5007 \AA$, the $\mathrm{H}_{2}$, and the $\mathrm{H}_{\mathrm{I}} 21 \mathrm{~cm}$ spectral lines together. The $\mathrm{H}_{2}$ profile in Fig. 9 is the stacked profile of the $\mathrm{H}_{2} 1.957 \mu \mathrm{m}$ and $\mathrm{H}_{2} \quad 1.835 \mu \mathrm{m}$ lines and appears to be slightly blueshifted with respect to the systemic velocity of the host galaxy. Its peak coincides with the peak of the blueshifted narrow component of the ionized gas and does not show clear signs of kinematically disturbed gas, but because of the low $\mathrm{S} / \mathrm{N}$ and the fluctuations of the continuum, we cannot completely rule out that there might be outflowing gas. 

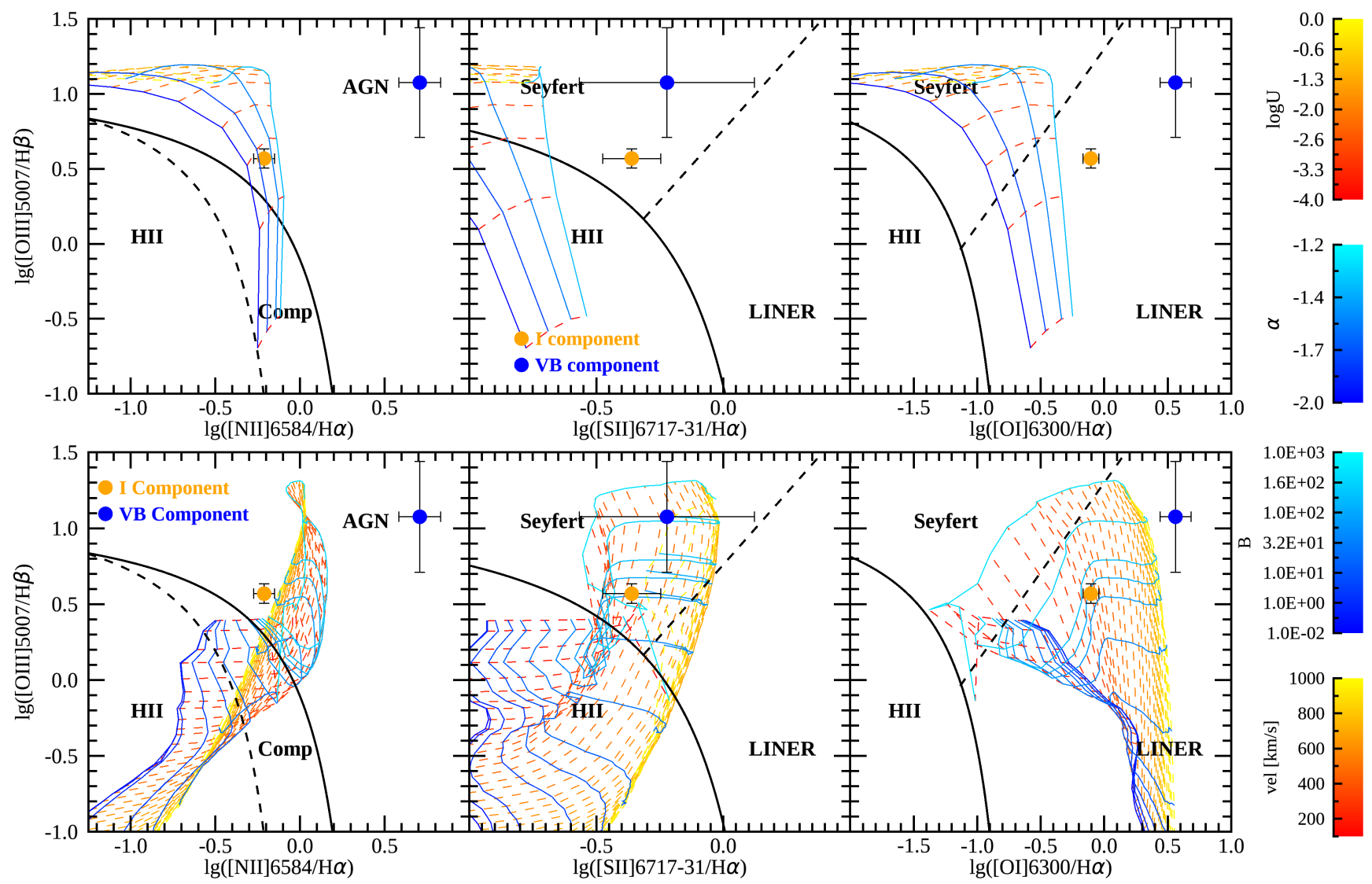

Fig. 7. BPT diagrams for the intermediate and very broad components with models of AGN photoionization (upper panels) and shocks (lower panels) with solar metallicity. The AGN photionization models have a gas density $n_{\mathrm{e}}=10^{5} \mathrm{~cm}^{-3}$, and the $\alpha$ and $\log U$ parameters vary in the same range as described in Fig. 6. The shock models have a pre-shock gas density of $n_{\mathrm{e}}=10^{3} \mathrm{~cm}^{-3}$, the dashed lines indicate models with constant shock velocity $v_{\mathrm{s}}$ (ranging in the interval $100-1000 \mathrm{~km} \mathrm{~s}^{-1}$, from left to right), and the solid lines refer to models with a constant magnetic parameter $B$ (ranging in the interval $0.01-1000 \mu \mathrm{G}$, from bottom to top). The solid and dashed black lines are the same as in Fig. 6.

HI gas has been detected in absorption against the radio continuum source by Véron-Cetty et al. (2000) and is shown inverted to emission for easy comparison in Fig. 9. These observations trace only the kinematics of gas that is located, in projection, in front of the compact radio source. The $\mathrm{H}_{\mathrm{I}}$ has a velocity shift $\left(v=116 \mathrm{~km} \mathrm{~s}^{-1}\right)$, which is comparable to the redshifted narrow component of the warm ionized gas and is characterized by a very narrow profile $\left(F W H M=18.8 \mathrm{~km} \mathrm{~s}^{-1}\right)$. This indicates that it might be connected to infalling clouds of atomic gas located in front of the radio source (as in the case of PKS B1718-649, see Maccagni et al. 2014).

A more global view on the atomic gas phase of the ISM could be obtained by using the absorption lines of the Na I D doublet at $\lambda \lambda 5890,5896 \AA$ (see, e.g., Lehnert et al. 2011). After the stellar continuum of the host galaxy was subtracted, we did not find evidence for the Na I D absorption in the nuclear spectrum of PKS B1934-63. This might be due to the compactness of the neutral ISM, which is concentrated in the inner regions of the galaxy (like the warm ionized gas) and does not absorb its diffuse starlight.

In the UVB part of the spectrum, we detected the ISM absorption features of the $\mathrm{Mg}$ II $\lambda \lambda 2795,2802 \AA$ doublet. These absorption lines trace gas in a low-ionization state where the $\mathrm{HI}$ is the dominant phase, and are superimposed on the $\mathrm{Mg}_{\mathrm{II}}$ emission lines at the same wavelengths because of the AGN. Even though we fit the $\mathrm{Mg}$ II lines with a simple model, which might ignore the complex emission line profile underneath the absorption (see Fig. A.2), we did not observe clear signs of kinematically disturbed gas in absorption. The $\mathrm{Mg}$ II emission and absorption were fit by single-Gaussian components with an $F W H M \sim 1250 \mathrm{~km} \mathrm{~s}^{-1}$ and $\sim 200 \mathrm{~km} \mathrm{~s}^{-1}$, respectively, and the absorption component is centered at the systemic redshift of the galaxy (see Appendix A for further details).

We detected such deep $\mathrm{Mg}_{\text {II }}$ absorption, which implies a covering factor of the line-emitting gas close to 1 , but no clear evidence for ISM absorption of the stellar continuum at the same wavelength (cf. Ca II, Na I D). This provides further evidence that the emission lines are emitted by a region that is compact relative to the stellar body of the galaxy.

We conclude that while we clearly detect an outflow of warm ionized gas, there is no strong evidence of cold outflowing gas traced by the $\mathrm{H}_{2}, \mathrm{HI}_{\mathrm{I}}$, and $\mathrm{Mg}$ II lines. This is in contrast with compact steep-spectrum radio sources such as IC 5063 (Tadhunter et al. 2014; Morganti et al. 2015; Oosterloo et al. 2017), PKS B1345+12 (Morganti et al. 2013; Dasyra \& Combes 2012), and 3C 305 (Morganti et al. 2005a), in which ionized gas outflows have also been detected. In these sources, the cold molecular $(\mathrm{CO})$ and warm molecular $\left(\mathrm{H}_{2}\right)$ gas has been found to be the dominant outflowing phase in terms of mass.

In recent years, the scenario in which cold gas is formed in situ, within the material swept away by the AGN, has gained more acceptance and has also been invoked to explain the 


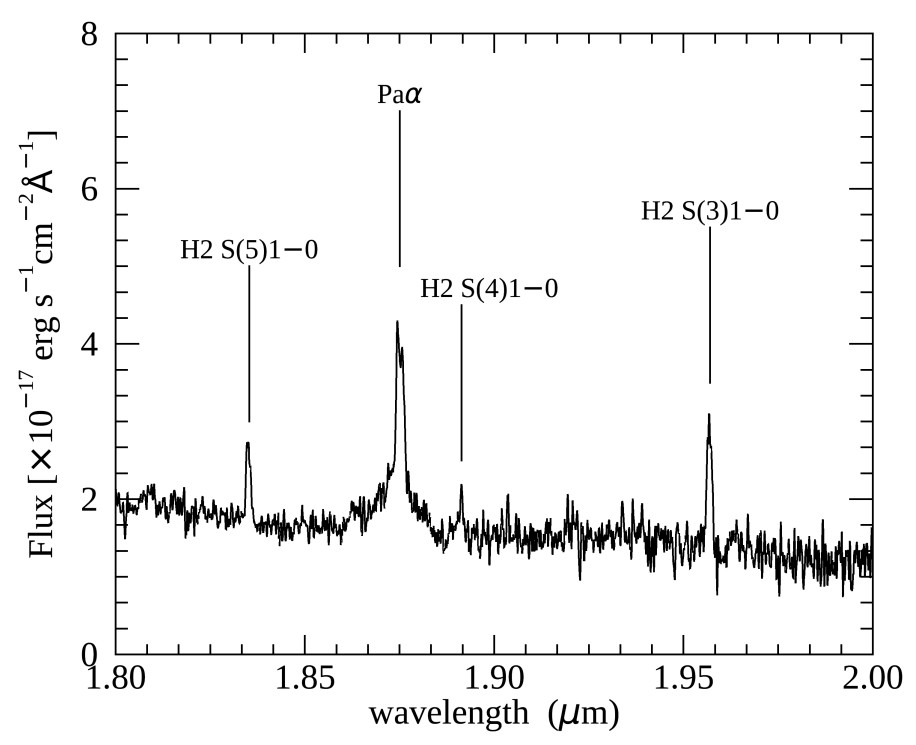

Fig. 8. Section of the nuclear spectrum of PKS B1934-63 in the NIR band showing the warm molecular $\mathrm{H}_{2}$ emission lines and the $\mathrm{Pa} \alpha$ line. The wavelength and the name of each line are indicated.

properties of the multi-phase outflow of compact steep-spectrum radio sources (Tadhunter et al. 2014; Morganti et al. 2015). According to the latter scenario, the AGN at first drives fast shocks into the ISM, ionizing the gas and heating it to high temperatures (higher than $10^{6} \mathrm{~K}$ ). The post-shock gas then cools down, accumulating as atomic and eventually cold molecular gas at a later stage. This means that outflows of warm ionized gas should be detected in the early phase of an AGN-ISM interaction, and only when sufficient time has elapsed for a substantial amount of gas to cool would we expect to be able to detect the cold molecular counterpart of these outflows (e.g., using CO lines). Recent simulations confirmed this scenario, finding that molecular gas starts to form around a few $10^{5} \mathrm{yr}$ from the start of the AGN-ISM interaction (Richings \& Faucher-Giguere 2017).

We performed a first attempt to test this scenario by integrating our findings with the outflow properties reported in the literature for other young compact radio sources. Compact radio sources have radio ages between $10^{2} \mathrm{yr}$ and $10^{5} \mathrm{yr}$ (Murgia 2003; Giroletti \& Polatidis 2009), and we used them to sample outflowing gas at different times. When we exclude PKS B193463 , our target, only a few other compact young radio sources have both an estimated radio age and observations of their multiphase ISM. It is worth mentioning that the sources we selected from the literature cover three orders of magnitudes in radio power, and their outflows properties might be due to an intrinsically different type of interaction between the ISM and the radio plasma.

PKS B1718-649 is a younger (kinematical age $\sim 10^{2} \mathrm{yr}$, Giroletti \& Polatidis 2009) compact radio source than PKS B1934-63, showing hints of warm ionized outflowing gas (blueshifted wings in the forbidden emission lines, see the optical spectrum in Filippenko 1985). Multi-wavelength observations find that as for our target, no evidence of outflowing gas in colder phases has been obtained for this source (atomic $\mathrm{HI}$, Maccagni et al. 2014; warm molecular $\mathrm{H}_{2}$, Maccagni et al. 2016; cold molecular CO, Maccagni et al. 2018).

An older compact radio source that is able to probe a more evolved stage of the AGN-ISM interaction is 3C 305. It has an estimated radiative age of $1.5 \times 10^{5} \mathrm{yr}$ (Murgia et al. 1999) and shows evidence of an outflow in the warm and cold molecular

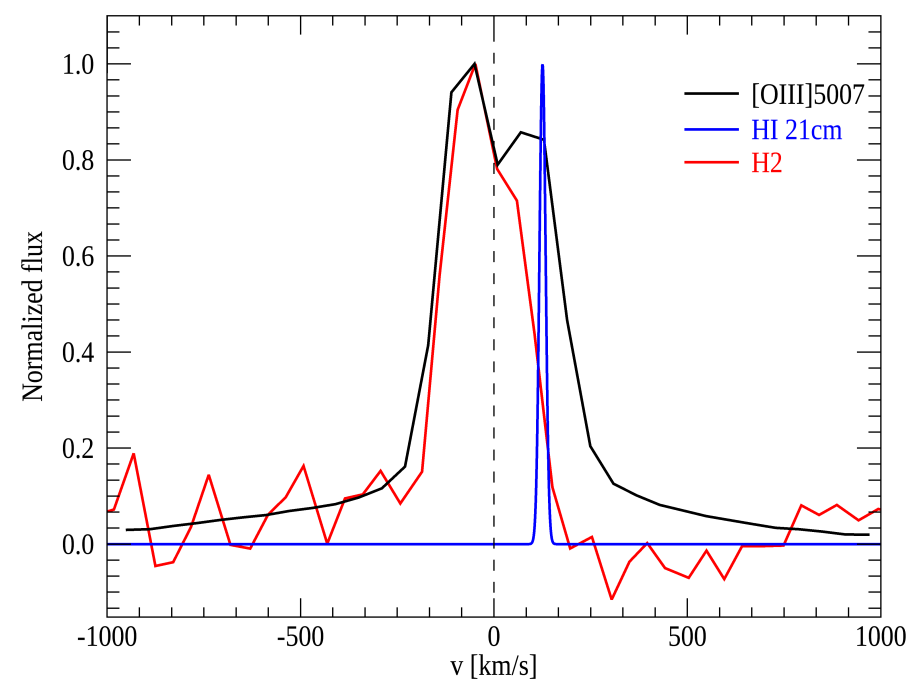

Fig. 9. Normalized $[\mathrm{O}$ III] $] \lambda 5007 \AA$ line (solid black), stacked $\mathrm{H}_{2}$ line (solid red), and $\mathrm{H}_{\mathrm{I}} 21 \mathrm{~cm}$ line (solid blue) in velocity space. Each line is normalized with respect to its maximum value. The zero velocity along the $\mathrm{x}$-axis is the systemic velocity of the galaxy as extracted in Sect. 3.1 and is marked with the black vertical dashed line. The $\mathrm{H}$ I line has been observed in absorption by Véron-Cetty et al. (2000) and is reproduced as an emission line using a Gaussian function and the parameters reported in the text.

gas (Guillard et al. 2012, and in prep.), and in the atomic $\mathrm{H}_{\mathrm{I}}$ and warm ionized gas (Morganti et al. 2005a).

Finally, B2 $0258+35$ is one of the oldest compact radio sources (radiative age $\leq 9 \times 10^{5} \mathrm{yr}$, Giroletti et al. 2005; $\leq 5 \times 10^{5} \mathrm{yr}$, Brienza et al., in prep.). Cold molecular CO and atomic $\mathrm{H}_{\mathrm{I}}$ gas have been detected (Prandoni et al. 2007; Struve et al. 2010) and show signs of disturbed kinematics suggestive of outflowing gas (Murthy et al., in prep.), while there is no evidence of outflowing gas in the warm ionized phase (see the optical spectrum presented in Emonts 2006, and the public data from the CALIFA survey ${ }^{1}$ ).

The time line presented in Fig. 10 summarizes all the information on these sources in the context of the scenario that we tested. The properties of the outflowing gas for PKS B193463 and of the compact steep-spectrum radio galaxies described above support the scenario in which cold molecular gas might form within the outflow material through cooling of shockheated gas. According to this scenario, PKS B1718-649 and PKS B1934-63 represent the earliest stages of the AGN-ISM interaction, when the outflowing gas is initially shock heated. This gas then starts to cool down in the post-shock region and can be detected as cold outflowing gas, as for 3C 305. Finally, the radio galaxy B2 $0258+35$ might be representative of the the final phases of the AGN-ISM interaction, when all the outflowing gas has completely cooled down. Considering the small amount of mass that is outflowing in the case of PKS B1934-63, most of the mass may currently be in a hotter phase (e.g., gas at $10^{7} \mathrm{~K}$ emitting in the X-ray band).

We are aware that the radiative and kinematical age of a radio galaxy might differ, and that the radiative age indicates the age of the particles within the radio lobes rather than the real age of the source. However, the ages that we used are the only available estimates, and it has been shown that the radiative age of 3C 305 is representative of the age of the radio source (Murgia et al. 1999).

1 http://califa.caha.es/ 


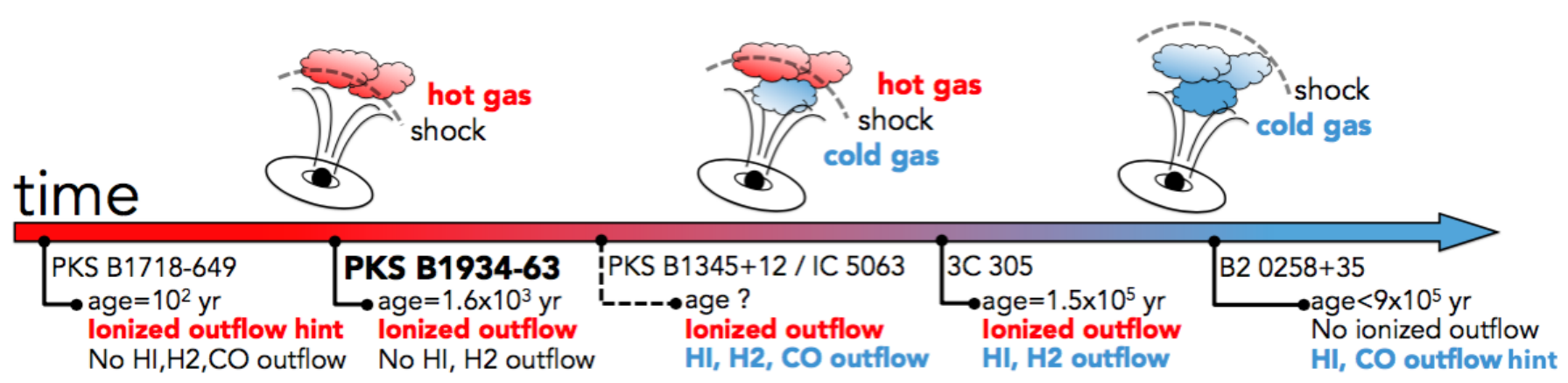

Fig. 10. Time-line sketch reporting the radio ages and the multi-phase properties of the outflows found in the compact sources PKS B1718-649, PKS B1934-63, PKS B1345+12, IC 5063, 3C 305, and B2 0258+35 in the context of the evolutionary scenario discussed in Sect. 7.

The time line in Fig. 10 also includes the two compact steep-spectrum radio sources PKS B1345+12 and IC 5063. These are two of the best examples of compact sources where extensive multi-wavelength studies found a multi-phase outflow (for PKS B1345+12 see Morganti et al. 2005b; Holt et al. 2011; Dasyra \& Combes 2012; for IC 5063 see Oosterloo et al. 2000; Morganti et al. 2007; Tadhunter et al. 2014; Morganti et al. 2015). Unfortunately, no reliable estimate of the age of these radio sources is available. However, considering the aforementioned studies, they might represent an intermediate stage of an AGNISM interaction, and this justifies their location in the time line presented in Fig. 10.

\section{Conclusions}

Compact and young radio galaxies are pivotal for our understanding of the feedback effect that radio sources have on their host galaxies. PKS B1934-63 is a young $\left(\sim 1.6 \times 10^{3} \mathrm{yr}\right)$ radio source, and given it proximity $(z=0.1824)$ and high radio power $\left(P_{1.4 \mathrm{GHz}}=10^{27.2} \mathrm{~W} \mathrm{~Hz}^{-1}\right)$, it is considered as an archetypal compact radio galaxy.

The nuclear spectrum of PKS B1934-63 shows doublepeaked gas emission lines that have broad wings, indicating a complex kinematics for the warm ionized gas and outflowing gas. The kinematical features of the intermediate and the very broad components, which are needed to model the broad part of the emission line profiles, and in particular their line width (FWHM of about 700 and $2000 \mathrm{~km} \mathrm{~s}^{-1}$, respectively), clearly reflect non-gravitational motions of gas that is connected to an AGN-driven outflow.

We find that about $6.8 \times 10^{6} M_{\odot}$ of warm ionized gas is concentrated in the inner $500 \mathrm{pc}$ of the host galaxy and only a small fraction of this gas is actually outflowing. Most of the warm ionized gas is included in a structure that shows a smooth velocity gradient in the velocity-position diagram presented in Fig. 5. Considering the rotating disk of warm ionized gas found by Roche et al. (2016) on larger scales, we tend to favor the hypothesis that this structure is a circum-nuclear disk with a radius of about $200 \mathrm{pc}$, which might constitute part of the gas reservoir from which the SMBH is fed (similar to what has been found, e.g., by Dumas et al. 2007; Hicks et al. 2013, and Maccagni et al. 2016).

The results of our spectro-astrometry study show that when we assume a biconical geometry for the outflow, the spatial extent of the broad wings of the [O III] $\lambda 5007 \AA$ line matches the diameter of the radio source (i.e., about $120 \mathrm{pc}$ ). This indicates that the outflow is likely driven by the expansion of the radio source jets, which is in line with what is commonly found in other compact and young radio sources (see, e.g., O'Dea et al. 2002; Holt et al. 2008; Tadhunter et al. 2014).

Using the density-diagnostic diagram introduced by Holt et al. (2011), we find a clear correlation between the FWHM of a component and its electron density (see Fig. 4), which is in line with the findings of Holt et al. (2011) and Rose et al. (2018). The warm ionized gas associated with the broad components reaches remarkably high electron densities $\left(10^{4.5}-10^{5.5} \mathrm{~cm}^{-3}\right)$. We attribute the broadening of the spectral lines to the interaction of the AGN with the ISM, and we argue that the FWHM-density relation that we find is mainly driven by the ability of the AGN-ISM interaction to compress the gas at different levels and increase its density.

Estimating the gas densities with the transauroral line technique allowed us a reliable estimate of the properties of the warm outflowing ionized gas. We obtain low-mass outflow rates (i.e., highest values in the range $10^{-3}-10^{-1} M_{\odot} \mathrm{yr}^{-1}$ ), and we find that only a small fraction of the available accretion power of the AGN is used to drive the outflow (i.e., maximum AGN feedback efficiency $F_{\text {kin }} \sim 10^{-3} \%$ ). This does not match the results of Fiore et al. (2017), for example, who reported higher average AGN feedback efficiencies in their collection of ionized gas outflows (i.e., 0.16-0.3\%). However, the latter outflows lack a robust estimate of the gas density, which is always taken to be $\leq 10^{3} \mathrm{~cm}^{-3}$, based on classical line ratio diagnostics or assumptions. It is worth mentioning that by adopting such densities, which are at least two order of magnitudes lower than the values we find, we would obtain an outflow mass rate and an AGN feedback efficiency that is compatible with the findings of Fiore et al. (2017). Instead, our results provide new evidence that AGN feedback occurs at a low efficiency, and are much close to the values reported by Villar-Martín et al. (2016) for luminous type II AGN and values by Rose et al. (2018), who used the same technique as we did here on a sample of local ULIRGs. This also highlights the importance of a fair comparison between the observed efficiencies and the cosmological models predictions (see Harrison et al. 2018, for a discussion).

Using the optical [S II $] \lambda \lambda 6717,31 / \mathrm{H} \alpha, \quad\left[\mathrm{O}_{\mathrm{I}}\right] \lambda 6300 / \mathrm{H} \alpha$, $\left[\mathrm{O}_{\text {III }}\right] \lambda \lambda 4958,5007 / 4363$, and $\mathrm{He}$ II $\lambda 4686 / \mathrm{H} \beta$ and the NIR $[\mathrm{Fe}$ II $] \lambda 1.257 \mu \mathrm{m} / \mathrm{Pa} \beta$ line-ratio diagnostics, we find that the AGN-ISM interaction drives shocks that heat the gas within the ISM. In particular, the comparison between model grids and the observed optical line ratios in the BPT diagrams indicates that the broad components of the warm ionized gas are ionized by fast shocks with velocities $v_{\mathrm{s}} \geq 400-500 \mathrm{~km} \mathrm{~s}^{-1}$, possibly reaching a few thousand $\mathrm{km} \mathrm{s}^{-1}$. These velocities are compatible 
with the width of the broad components and suggest that shocks are a feasible mechanism to accelerate the warm ionized gas to high velocities.

By studying the $\mathrm{Mg}$ II absorption and the $\mathrm{H}_{2}$ emission lines, we find that the absorbing low-ionization ISM and warm molecular gas do not show signs of outflowing and/or kinematically disturbed gas. In addition, past $\mathrm{H}_{\mathrm{I}}$ observations of the nuclear region of the host galaxy did not find outflowing gas in the atomic phase (Véron-Cetty et al. 2000). This is at odds with other compact, steep-spectrum radio sources that are known to host ionized gas outflows, which usually show massive cold gas outflows (Morganti et al. 2005a; Dasyra \& Combes 2012; Morganti et al. 2013, 2015; Tadhunter et al. 2014). By integrating our findings with information on other compact radio galaxies from the literature, we suggest that the lack of cold outflowing gas in PKS B1934-63 might be explained by the fact that the shock-ionized outflowing gas did not have enough time to cool down given the young age of the radio source, and thus, the fact that the AGN-ISM interaction occurred recently. It is also possible that a fraction of the outflowing gas is in a hotter phase (e.g., $T \sim 10^{7} \mathrm{~K}$ ) that is missed by our observations. Our results strengthen the hypothesis that in general the AGN drives shocks within the ISM during an AGN-ISM interaction, and cold gas is formed in situ within the outflowing material. New observations probing the cold molecular gas (i.e., CO) in PKS B1934-63 will be crucial for completing the picture on the presence and kinematics of the colder gas phase.

Our work shows that a systematic and detailed characterization of the multi-phase properties of the outflows in compact young radio galaxies offers the possibility to study the origin of cold gas within outflows. It also has the potential of shedding light on the relevance of AGN feedback operated by jets on galactic scales.

Acknowledgements. The authors thank the anonymous referee for the usefu comments that improved the quality of the paper. The research leading to these results has received funding from the European Research Council under the European Union's Seventh Framework Programme (FP/2007-2013) / ERC Advanced Grant RADIOLIFE-320745. Based on observations collected at the European Organisation for Astronomical Research in the Southern Hemisphere under ESO programme 87.B-0614A.

\section{References}

Allen, M. G., Groves, B. A., Dopita, M. A., Sutherland, R. S., \& Kewley, L. J 2008, ApJS, 178, 20

Axon, D. J., Capetti, A., Fanti, R., et al. 2000, AJ, 120, 2284

Bailey, J. 1998a, MNRAS, 301, 161

Bailey, J. A. 1998b, in Proc. SPIE, Vol. 3355, Optical Astronomical Instrumentation, ed. S. D'Odorico, 932

Baldwin, J. A., Phillips, M. M., \& Terlevich, R. 1981, PASP, 93, 5

Batcheldor, D., Tadhunter, C., Holt, J., et al. 2007, ApJ, 661, 70

Benson, A. J., Bower, R. G., Frenk, C. S., et al. 2003, ApJ, 599, 38

Best, P. N., \& Heckman, T. M. 2012, MNRAS, 421, 1569

Bicknell, G. V., Dopita, M. A., \& O’Dea, C. P. O. 1997, ApJ, 485, 112

Binette, L., Wilson, A. S., \& Storchi-Bergmann, T. 1996, A\&A, 312, 365

Blietz, M., Cameron, M., Drapatz, S., et al. 1994, ApJ, 421, 92

Bolton, J. G., Gardner, F. F., \& Mackey, M. B. 1963, Nature, 199, 682

Bongiorno, A., Schulze, A., Merloni, A., et al. 2016, A\&A, 588, A78

Bower, R. G., Benson, A. J., Malbon, R., et al. 2006, MNRAS, 370, 645

Bruzual, G., \& Charlot, S. 2003, MNRAS, 344, 1000

Callingham, J. R., Gaensler, B. M., Ekers, R. D., et al. 2015, ApJ, 809, 168

Calzetti, D., Armus, L., Bohlin, R. C., et al. 2000, ApJ, 533, 682

Cid Fernandes, R., Mateus, A., Sodré, L., Stasińska, G., \& Gomes, J. M. 2005, MNRAS, 358, 363

Cimatti, A., Dey, A., van Breugel, W., Hurt, T., \& Antonucci, R. 1997, ApJ, 476, 677

Comerford, J. M., Gerke, B. F., Newman, J. A., et al. 2009, ApJ, 698, 956

Couto, G. S., Storchi-Bergmann, T., Axon, D. J., et al. 2013, MNRAS, 435, 2982
Couto, G. S., Storchi-Bergmann, T., \& Schnorr-Müller, A. 2017, MNRAS, 469, 1573

Dasyra, K. M., \& Combes, F. 2012, A\&A, 541, L7

de Vries, W. H., Barthel, P. D., \& O’Dea, C. P. 1997a, A\&A, 321, 105

de Vries, W. H., O’Dea, C. P., Baum, S. A., et al. 1997b, ApJS, 110, 191

Di Matteo, T., Springel, V., \& Hernquist, L. 2005, Nature, 433, 604

di Serego Alighieri, S., Field, G. B., \& Cimatti, A. 1995, in The Physics of the Interstellar Medium and Intergalactic Medium, eds. A. Ferrara, C. F. McKee, C. Heiles, \& P. R. Shapiro, ASP Conf. Ser., 80, 276

Dumas, G., Mundell, C. G., Emsellem, E., \& Nagar, N. M. 2007, MNRAS, 379, 1249

Emonts, B. H. C. 2006, PhD thesis, University of Groningen

Fabian, A. C. 1999, MNRAS, 308, L39

Fanti, C., \& Fanti, R. 1994, in The Physics of Active Galaxies, eds. G. V. Bicknell, M. A. Dopita, \& P. J. Quinn, ASP Conf. Ser., 54, 341

Fanti, R., Fanti, C., Schilizzi, R. T., et al. 1990, A\&A, 231, 333

Ferland, G. J., Porter, R. L., van Hoof, P. A. M., et al. 2013, Rev. Mex. Astron. Astrofis., 49, 137

Filippenko, A. V. 1985, AJ, 90, 1172

Fiore, F., Feruglio, C., Shankar, F., et al. 2017, A\&A, 601, A143

Forbes, D. A., \& Ward, M. J. 1993, ApJ, 416, 150

Fosbury, R. A. E., Bird, M. C., Nicholson, W., \& Wall, J. V. 1987, MNRAS, 225, 761

García-Burillo, S., Combes, F., Ramos Almeida, C., et al. 2016, ApJ, 823, L12

Geréb, K., Maccagni, F. M., Morganti, R., \& Oosterloo, T. A. 2015a, A\&A, 575, A44

Geréb, K., Morganti, R., Oosterloo, T. A., Hoppmann, L., \& Staveley-Smith, L. 2015b, A\&A, 580, A43

Giroletti, M., Giovannini, G., \& Taylor, G. B. 2005, A\&A, 441, 89

Giroletti, M., \& Polatidis, A. 2009, Astron. Nachr., 330, 193

Granato, G. L., De Zotti, G., Silva, L., Bressan, A., \& Danese, L. 2004, ApJ, 600,580

Groves, B. A., \& Allen, M. G. 2010, New Astron., 15, 614

Guillard, P., Ogle, P. M., Emonts, B. H. C., et al. 2012, ApJ, 747, 95

Harrison, C. M., Costa, T., Tadhunter, C. N., et al. 2018, Nat. Astron., 2, 198

Heckman, T. M., Kauffmann, G., Brinchmann, J., et al. 2004, ApJ, 613, 109

Heckman, T. M., Smith, E. P., Baum, S. A., et al. 1986, ApJ, 311, 526

Hicks, E. K. S., Davies, R. I., Maciejewski, W., et al. 2013, ApJ, 768, 107

Holt, J., Tadhunter, C., Morganti, R., et al. 2006, MNRAS, 370, 1633

Holt, J., Tadhunter, C. N., \& Morganti, R. 2008, MNRAS, 387, 639

Holt, J., Tadhunter, C. N., Morganti, R., \& Emonts, B. H. C. 2011, MNRAS, 410,1527

Hopkins, P. F., \& Elvis, M. 2010, MNRAS, 401, 7

Inskip, K. J., Tadhunter, C. N., Morganti, R., et al. 2010, MNRAS, 407, 1739

Kauffmann, G., Heckman, T. M., Tremonti, C., et al. 2003, MNRAS, 346, 1055

Kewley, L., Groves, B., Kauffmann, G., \& Heckman, T. 2006, MNRAS, 372, 961

Kewley, L. J., Heisler, C. A., Dopita, M. A., \& Lumsden, S. 2001, ApJS, 132, 37

King, A. 2003, ApJ, 596, L27

Kormendy, J., \& Ho, L. C. 2013, ARA\&A, 51, 511

Labiano, A. 2008, A\&A, 488, L59

Lamastra, A., Bianchi, S., Matt, G., et al. 2009, A\&A, 504, 73

Lehnert, M. D., Tasse, C., Nesvadba, N. P. H., Best, P. N., \& van Driel, W. 2011, A\&A, 532, L3

Maccagni, F. M., Morganti, R., Oosterloo, T. A., \& Mahony, E. K. 2014, A\&A, 571, A67

Maccagni, F. M., Santoro, F., Morganti, R., et al. 2016, A\&A, 588, A46

Maccagni, F. M., Morganti, R., Oosterloo, T. A., Oonk, J. B. R., \& Emonts, B. H. C. 2018, A\&A, 614, A42

Markwardt, C. B. 2009, in Astronomical Data Analysis Software and Systems XVIII, eds. D. A. Bohlender, D. Durand, \& P. Dowler, ASP Conf. Ser., 411, 251

Matsuoka, K., Nagao, T., Maiolino, R., et al. 2017, A\&A, 608, A90

Momcheva, I. G., Lee, J. C., Ly, C., et al. 2013, AJ, 145, 47

Morganti, R., Fogasy, J., Paragi, Z., Oosterloo, T., \& Orienti, M. 2013, Science, 341,1082

Morganti, R., Holt, J., Saripalli, L., Oosterloo, T. A., \& Tadhunter, C. N. 2007, A\&A, 476, 735

Morganti, R., Oosterloo, T., Oonk, J. B. R., Frieswijk, W., \& Tadhunter, C. 2015, A\&A, 580, A1

Morganti, R., Oosterloo, T. A., Tadhunter, C. N., van Moorsel, G., \& Emonts, B. 2005a, A\&A, 439, 521

Morganti, R., Tadhunter, C. N., \& Oosterloo, T. A. 2005b, A\&A, 444, L9

Murgia, M. 2003, PASA, 20, 19

Murgia, M., Fanti, C., Fanti, R., et al. 1999, A\&A, 345, 769

O’Dea, C. P., de Vries, W. H., Koekemoer, A. M., et al. 2002, AJ, 123, 2333

Ojha, R., Fey, A. L., Johnston, K. J., et al. 2004, AJ, 127, 1977

Oosterloo, T. A., Morganti, R., Tzioumis, A., et al. 2000, AJ, 119, 2085 
F. Santoro et al.: Probing multi-phase outflows and AGN feedback in compact radio galaxies: the case of PKS B1934-63

Oosterloo, T., Raymond Oonk, J. B., Morganti, R., et al. 2017, A\&A, 608, A38 Orienti, M., \& Dallacasa, D. 2008, A\&A, 487, 885

Osterbrock, D. E., \& Ferland, G. J. 2006, Astrophysics of gaseous nebulae and active galactic nuclei (Sausalito: University Science Books)

Prandoni, I., Laing, R. A., Parma, P., et al. 2007, in From Z-Machines to ALMA (Sub)Millimeter Spectroscopy of Galaxies, eds. A. J. Baker, J. Glenn, A. I. Harris, J. G. Mangum, \& M. S. Yun, ASP Conf. Ser., 271

Ramos Almeida, C., Tadhunter, C. N., Inskip, K. J., et al. 2011, MNRAS, 410, 1550

Richings, A. J., \& Faucher-Giguere, C.-A. 2017, MNRAS, 474, 3673

Roche, N., Humphrey, A., Lagos, P., et al. 2016, MNRAS, 459, 4259

Rodríguez-Ardila, A., Pastoriza, M. G., Viegas, S., Sigut, T. A. A., \& Pradhan, A. K. 2004, A\&A, 425, 457

Rose, M., Tadhunter, C., Ramos Almeida, C., et al. 2018, MNRAS, 474, 128

Silk, J., \& Rees, M. J. 1998, A\&A, 331, L1

Sivjee, G. G., Romick, G. J., \& Rees, M. H. 1979, ApJ, 229, 432

Springel, V., Di Matteo, T., \& Hernquist, L. 2005, MNRAS, 361, 776

Storchi-Bergmann, T., Winge, C., Ward, M. J., \& Wilson, A. S. 1999, MNRAS, 304,35

Struve, C., Oosterloo, T., Sancisi, R., Morganti, R., \& Emonts, B. H. C. 2010, A\&A, 523, A75

Sutherland, R., Dopita, M., Binette, L., \& Groves, B. 2013, MAPPINGS III: Modelling And Prediction in Photolonized Nebulae and Gasdynamical Shocks, Astrophysics Source Code Library
Sutherland, R. S., \& Dopita, M. A. 2017, ApJS, 229, 34

Tadhunter, C. 2016, Astron. Nachr., 337, 159

Tadhunter, C. N., Metz, S., \& Robinson, A. 1994a, MNRAS, 268, 989

Tadhunter, C. N., Shaw, M. A., \& Morganti, R. 1994b, MNRAS, 271, 807

Tadhunter, C., Wills, K., Morganti, R., Oosterloo, T., \& Dickson, R. 2001, MNRAS, 327, 227

Tadhunter, C., Morganti, R., Rose, M., Oonk, J. B. R., \& Oosterloo, T. 2014, Nature, 511, 440

Takami, M., Bailey, J., \& Chrysostomou, A. 2003, A\&A, 397, 675

Tingay, S. J., Macquart, J.-P., Collier, J. D., et al. 2015, AJ, 149, 74

Tzioumis, A. K., Jauncey, D. L., Preston, R. A., et al. 1989, AJ, 98, 36

Vernet, J., Dekker, H., D’Odorico, S., et al. 2011, A\&A, 536, A105

Véron-Cetty, M.-P., Woltjer, L., Staveley-Smith, L., \& Ekers, R. D. 2000, A\&A, 362,426

Villar-Martín, M., Tadhunter, C., Morganti, R., Axon, D., \& Koekemoer, A. 1999, MNRAS, 307, 24

Villar-Martín, M., Alonso-Herrero, A., di Serego Alighieri, S., \& Vernet, J. 2000, A\&AS, 147, 291

Villar-Martín, M., Arribas, S., Emonts, B., et al. 2016, MNRAS, 460, 130

Wagner, A. Y., Bicknell, G. V., \& Umemura, M. 2012, ApJ, 757, 136

Wagner, A. Y., Bicknell, G. V., Umemura, M., Sutherland, R. S., \& Silk, J. 2016, Astron. Nachr., 337, 167

Zakamska, N. L., Strauss, M. A., Krolik, J. H., et al. 2003, AJ, 126, 2125

Zheng, W. 1988, Astrophys. Lett. Commun., 27, 275 


\section{Appendix A: Stellar population and line fitting}

This appendix includes the results of the stellar population and emission line modeling. In the following we describe the fitting procedure for some of the spectral lines in more detail.

In the case of the $\operatorname{Mg}_{\text {II }} \lambda \lambda 2795,2802 \AA$ doublet, the ISM $\mathrm{Mg}$ II absorption lines are superimposed on the emission lines by the AGN light. For each line of the doublet we used a Gaussian function to model the AGN emission and a Gaussian function to model the ISM absorption (see Fig. A.2). The separation between the two emission and absorption components was fixed according to the laboratory rest wavelengths of the doublet. In addition, we fixed their sigma to be the same. We find that the $\mathrm{Mg}_{\text {II }}$ emission is fit by a Gaussian with an $F W H M=1245 \pm 99 \mathrm{~km} \mathrm{~s}^{-1}$ and a velocity shift $v=-53 \pm 35 \mathrm{~km} \mathrm{~s}^{-1}$, while the absorption component has an $F W H M=200 \pm 38 \mathrm{~km} \mathrm{~s}^{-1}$ and a velocity shift $v=14 \pm 36 \mathrm{~km} \mathrm{~s}^{-1}$.

In the case of the $\left[\mathrm{N}\right.$ II] and $\left[\mathrm{O}_{\mathrm{I}}\right]$ lines, we fixes the $\left[\mathrm{N}_{\text {II }}\right] \lambda 6584 / \lambda 6548$ and the $\left[\mathrm{O}_{\mathrm{I}}\right] \lambda 6300 / \lambda 6363$ line ratios to be equal to 3 according to atomic physics (Osterbrock \& Ferland 2006). The $\left[\mathrm{O}_{\mathrm{II}}\right] \lambda 7319 / \lambda 7330$ line ratio was fixed to be 1.24 because it was found not to vary with density. The $\left[\mathrm{O}_{\text {II }}\right] \lambda 3729 / \lambda 3726$ and the $\left[\mathrm{S}_{\text {II }}\right] \lambda 6717 / \lambda 6731$ ratios were limited within their theoretical values at low and high densities (Osterbrock \& Ferland 2006). We also limited the
[S II] $\lambda 4069 / \lambda 4076$ ratio to be in the range 3.01-3.28 according to the calculation made by Rose et al. (2018) at different densities.

The [O I $] \lambda 6300 \AA$ line shows a different profile compared to all the other forbidden emission lines, which might be due to contamination from the $[\mathrm{S}$ III] $\lambda 6312 \AA$ line. Considering the flux of the $\left[\mathrm{S}_{\text {III }}\right] \lambda 9531 \AA$ emission line, we can predict the minimum total flux and peak emission expected for the [S III] $] 6312 \AA$ line. To do this, we assumed the lower value that the [S III] $9531 / 6312$ line ratio reaches in the high temperature regime (see Osterbrock \& Ferland 2006). We find that we expect to detect the emission of the $\left[\mathrm{S}_{\mathrm{III}}\right] \lambda 6312 \AA$ line in the nuclear spectrum of PKS B1934-63. We predict that the [S III] $] 6312 \AA$ line has a total flux higher than $17.5 \times 10^{-17} \mathrm{erg} \mathrm{s}^{-1} \mathrm{~cm}^{-2}$ and a line peak (assuming the four kinematical components of the [O III] model) greater than about $3 \times 10^{-17} \mathrm{erg} \mathrm{s}^{-1} \mathrm{~cm}^{-2} \AA^{-1}$. For this reason, when we fit the $\left[\mathrm{O}_{\mathrm{I}}\right] \lambda \lambda 6300-63 \AA$ doublet, we also included an [S III] $\lambda 6312 \AA$ component that is well reproduced using only the intermediate component of the [O III] model (see Fig. A.8).

In the fit of the [O II] $\lambda \lambda 7319,30 \AA$ we also introduced an additional Gaussian component to take into account the emission of the [O $\left.{ }_{\text {II }}\right] \lambda 7381 \AA$ emission in the red wing of the doublet. In the same way, we take into account the emission of the $\mathrm{H}_{2}$ $\mathrm{S}(4) 1-0$ line in the fit of the $\mathrm{Pa} \alpha$ line profile.

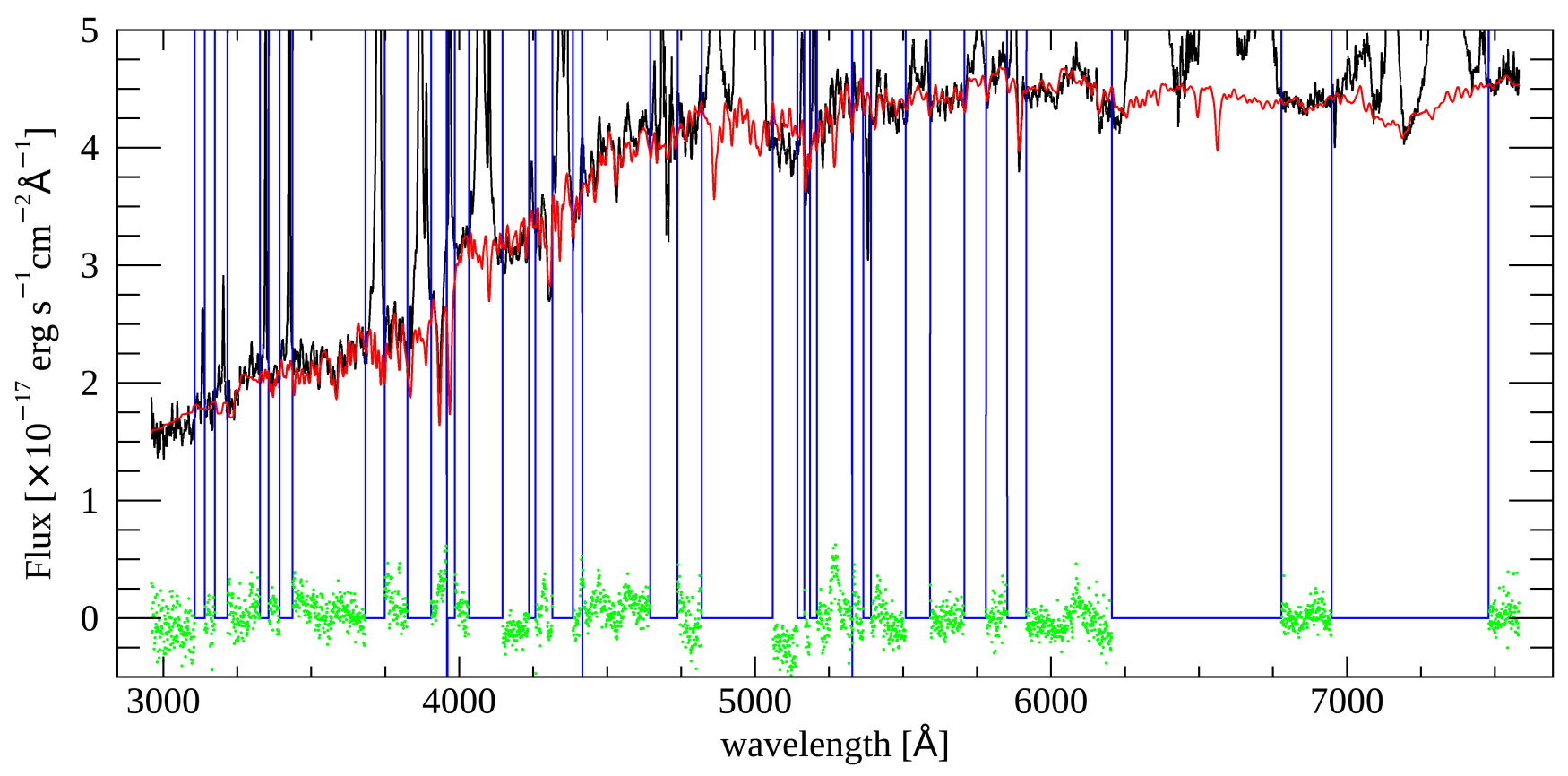

Fig. A.1. Nuclear spectrum (black solid line) and best-fit model (red solid line) for the continuum emission and residual (green points). The masked regions that correspond to the emission lines are indicated in blue. The observed spectrum is smoothed with a box of $3 \AA$. 
Table A.1. Fluxes of the four kinematical components of each fitted line.

\begin{tabular}{|c|c|c|c|c|c|}
\hline Line ID & $\lambda(\AA)$ & Flux $_{1 \mathrm{~N}}$ & Flux $_{2 \mathrm{~N}}$ & Flux $_{\mathrm{I}}$ & Flux $_{\mathrm{VB}}$ \\
\hline [O II]3726 & 3726.03 & 34.7 & 46.8 & 91.1 & 28.7 \\
\hline [O II]3729 & 3728.82 & 36.9 & 48.1 & 39.9 & 7.10 \\
\hline [S II $] 4068$ & 4068.60 & 1.40 & 2.50 & 52.0 & 34.0 \\
\hline$\left[\mathrm{S}_{\text {II }}\right] 4076$ & 4076.35 & 0.42 & 0.84 & 17.0 & 11.0 \\
\hline \multirow[t]{2}{*}{$\mathrm{H} \gamma$} & 4340.50 & 6.95 & 6.20 & 27.6 & 18.1 \\
\hline & & 7.23 & 6.41 & 24.3 & $9.51 \pm 2.56$ \\
\hline \multirow[t]{2}{*}{ [O III]4636 } & 4363.20 & 5.80 & 3.94 & 10.5 & 22.3 \\
\hline & & 5.48 & 3.68 & 14.7 & - \\
\hline He II4686 & 4685.7 & 5.41 & 4.90 & $4.19 \pm 0.74$ & - \\
\hline $\mathrm{H} \beta$ & 4861.33 & 21.9 & 24.1 & 59.0 & $16.6 \pm 7.6$ \\
\hline [O III] $] 5007$ & 5006.84 & 297 & 297 & 218 & 197 \\
\hline [O I $] 6300$ & 6300.30 & 54.4 & 65.2 & 240 & 211 \\
\hline [S III]6312 & 6312.10 & - & - & 34.0 & - \\
\hline $\mathrm{H} \alpha$ & 6562.80 & $99.4 \pm 10.3$ & $106 \pm 11$ & $306 \pm 32$ & $58.0 \pm 10.1$ \\
\hline [N II $] 6584$ & 6583.41 & $95.7 \pm 10.1$ & $106 \pm 11$ & $188 \pm 20$ & $295 \pm 32$ \\
\hline$\left[\mathrm{S}_{\text {II }}\right] 6717$ & 6716.47 & 40.7 & 41.5 & 41.5 & $20.0 \pm 13.2$ \\
\hline [S II]6731 & 6730.85 & 36.7 & 39.9 & 92.1 & $14.7 \pm 5.46$ \\
\hline$\left[\mathrm{O}_{\text {II }}\right] 7318,19$ & 7320.10 & 4.07 & 5.03 & 51.8 & 92.7 \\
\hline$\left[\mathrm{O}_{\mathrm{II}}\right] 7330,31$ & 7330.20 & 3.28 & 4.05 & 41.7 & 74.7 \\
\hline [S III $] 9531$ & 9530.6 & 59.0 & 61.1 & 83.0 & $56.1 \pm 7.0$ \\
\hline $\mathrm{Pa} \alpha$ & 18751.01 & 17.7 & 18.0 & 29.0 & 66.8 \\
\hline
\end{tabular}

Notes. The fluxes are given in units of $10^{-17} \mathrm{erg} \mathrm{s}^{-1} \mathrm{~cm}^{-2}$. The first and second column indicate the emission line ID and rest frame wavelength, respectively. For the $\mathrm{H} \gamma$ and the [O $\mathrm{III}] \lambda 4363 \AA$ lines we report two sets of fluxes. These lines are blended, and the first row reports the fluxes obtained by modeling both lines with the [O $\mathrm{III}]$ model, while the second row reports the fluxes obtained by modeling the $\mathrm{H} \gamma$ line with the $\mathrm{H} \beta$ model and the $\left[\mathrm{O}_{\mathrm{III}}\right] \lambda 4363 \AA$ line with the [O III] model. When not indicated, the error on the reported flux is $10 \%$.

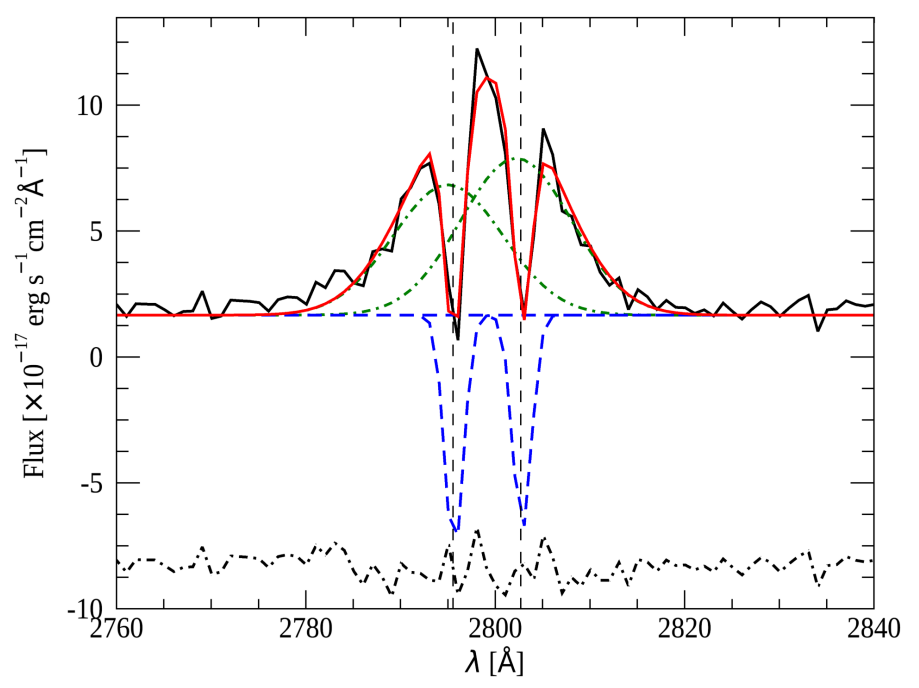

Fig. A.2. $\mathrm{Mg}$ II $\lambda \lambda 2795,2802 \AA$ lines (black solid line) and best-fit model (red solid line). Each line is fit with one Gaussian component to take into account the emission (green dot-dashed line) and one Gaussian component to take into account the absorption (blue dashed line). The residuals of the fit are normalized and plotted below the spectrum (black dotdashed line). The vertical dashed lines mark the restframe wavelength of the fitted emission lines.

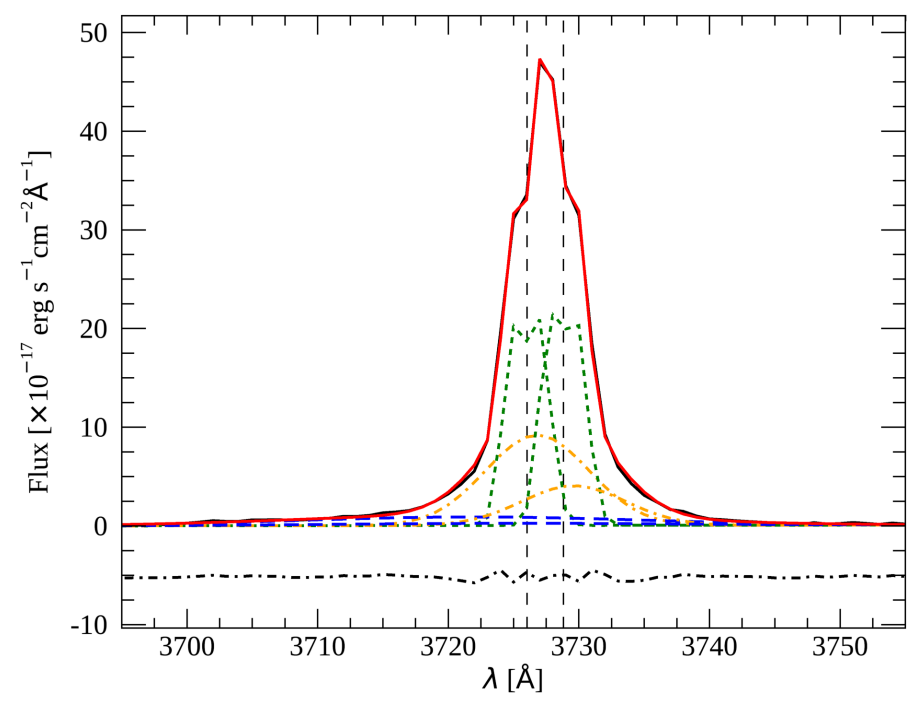

Fig. A.3. $\left[\mathrm{O}_{\mathrm{II}}\right] \lambda \lambda 3726,29 \AA$ emission lines (black solid line) and bestfit model (red solid line). Each line is modeled using the [O III] model, the $1 \mathrm{~N}$ and $2 \mathrm{~N}$ components are plotted together with the green dotted line, the I component is shown with the golden dot-dashed line, and the VB component with the blue dashed line. The residuals of the fit are normalized and plotted below the spectrum (black dot-dashed line). The vertical dashed lines mark the restframe wavelength of the fitted emission lines. 


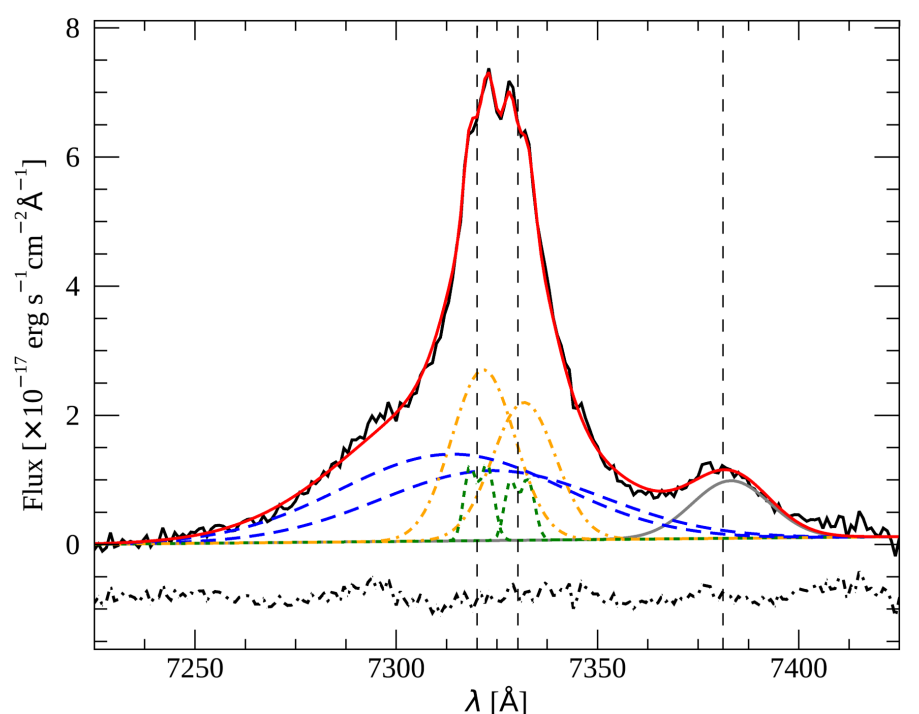

Fig. A.4. $\left[\mathrm{O}_{\mathrm{II}}\right] \lambda \lambda 7319,30 \AA$ and $\left[\mathrm{O}_{\mathrm{II}}\right] \lambda 7381 \AA$ emission lines (black solid line) and best-fit model (red solid line). Each line of the [O II $] \lambda \lambda 7320-30 \AA$ doublet is modeled using the [O III] model, the $1 \mathrm{~N}$ and $2 \mathrm{~N}$ components are plotted together with the green dotted line, the I component is shown with the golded dot-dashed line, and the VB component with the blue dashed line. The [O II] $] 73381 \AA$ line is modeled with a single Gaussian function indicated with the gray solid line. The residuals of the fit are normalized and plotted below the spectrum (black dot-dashed line). The vertical dashed lines mark the restframe wavelength of the fitted emission lines.

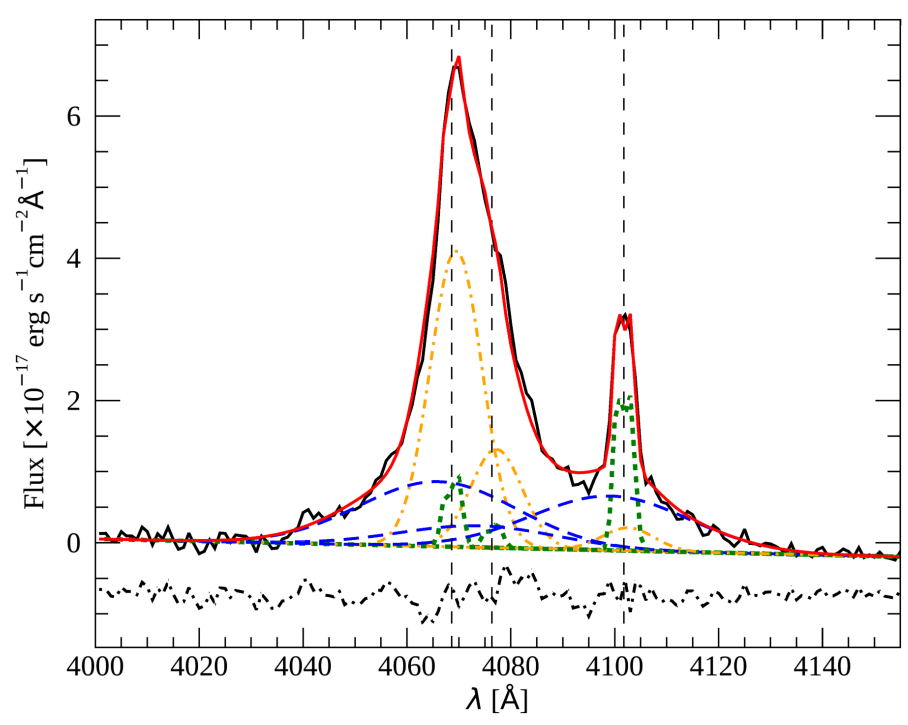

Fig. A.5. [S II] $] \lambda \lambda 4069,76 \AA$ and $\mathrm{H} \delta$ emission lines (black solid line) and best-fit model (red solid line). Each line is modeled using the [O III] model, the $1 \mathrm{~N}$ and $2 \mathrm{~N}$ components are plotted together with the green dotted line, the I component is shown with the golden dot-dashed line, and the VB component with the blue dashed line. The residuals of the fit are normalized and plotted below the spectrum (black dot-dashed line). The vertical dashed lines mark the restframe wavelength of the fitted emission lines.

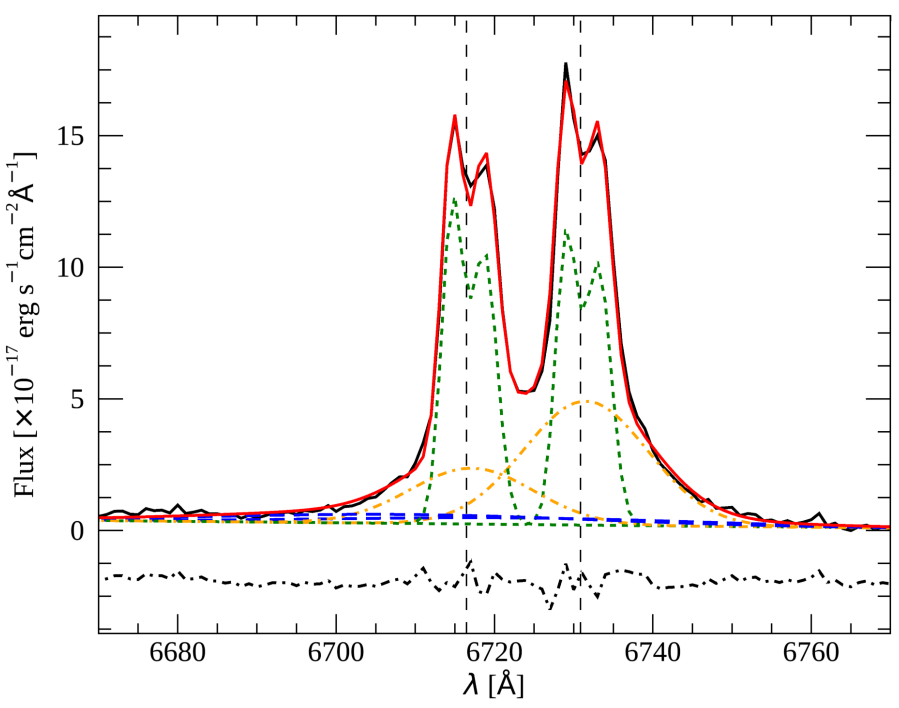

Fig. A.6. $[\mathrm{S}$ II $] \lambda \lambda 6717,31 \AA$ A emission lines (black solid line) and bestfit model (red solid line). Each line is modeled using the [O III] model, the $1 \mathrm{~N}$ and $2 \mathrm{~N}$ components are plotted together with the green dotted line, the I component is shown with the golden dot-dashed line, and the VB component with the blue dashed line. The residuals of the fit are normalized and plotted below the spectrum (black dot-dashed line). The vertical dashed lines mark the restframe wavelength of the fitted emission lines.

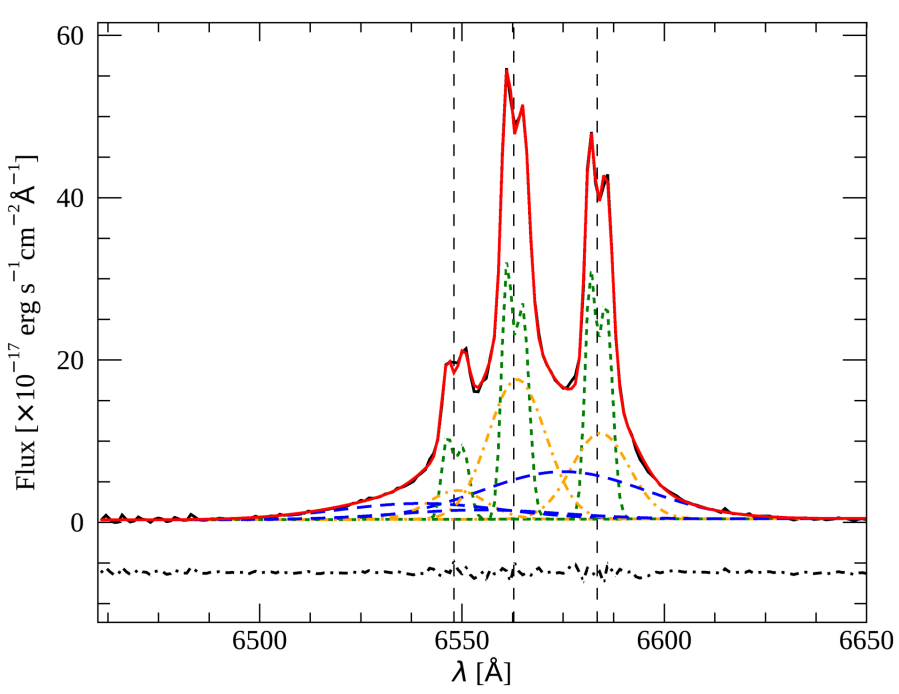

Fig. A.7. $\mathrm{H} \alpha$ and $[\mathrm{N}$ II] $] \lambda \lambda 6548-84 \AA$ emission lines (black solid line) and best-fit model (red solid line). Each line is modeled using the [O III] model, the $1 \mathrm{~N}$ and $2 \mathrm{~N}$ components are plotted together with the green dotted line, the I component is shown with the golded dot-dashed line, and the VB component with the blue dashed line. The residuals of the fit are normalized and plotted below the spectrum (black dot-dashed line). The vertical dashed lines mark the restframe wavelength of the fitted emission lines. 


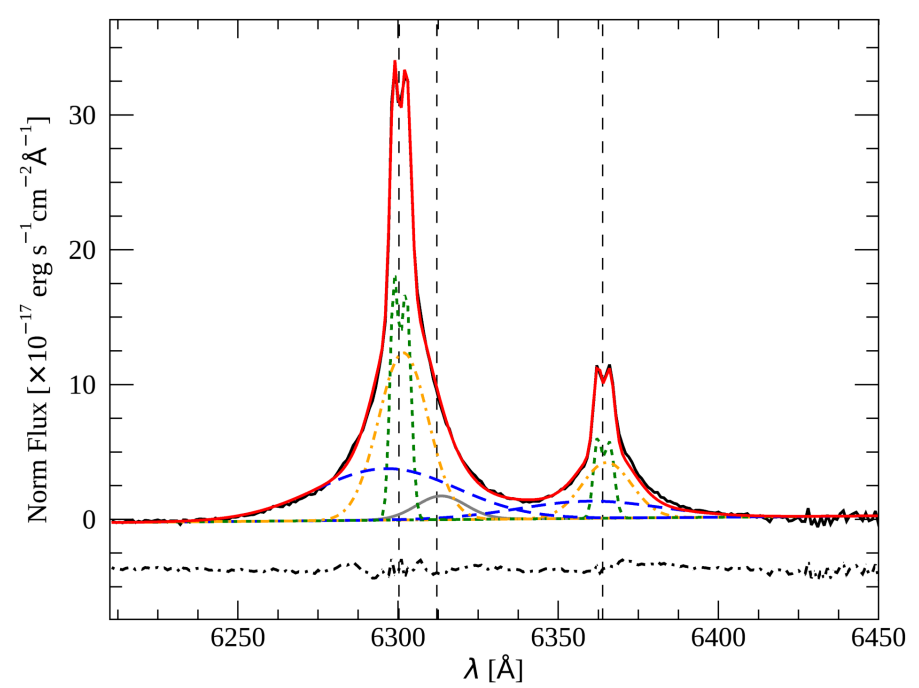

Fig. A.8. $\left[\mathrm{O}_{\mathrm{I}}\right] \lambda \lambda 6300-63 \AA$ and $[\mathrm{S}$ III] $] \lambda 6312 \AA$ emission lines (black solid line) and best-fit model (red solid line). Each line of the [O I] $\lambda \lambda 6300-63 \AA$ doublet is modeled using the [O III] model, the $1 \mathrm{~N}$ and $2 \mathrm{~N}$ components are plotted together with the green dotted line, the I component is shown with the golded dot-dashed line, and the VB component with the blue dashed line. The $[\mathrm{S} \mathrm{III]}] \lambda 6312 \AA$ line is modeled with a single Gaussian function indicated with the gray solid line. The residuals of the fit are normalized and plotted below the spectrum (black dot-dashed line). The vertical dashed lines mark the restframe wavelength of the fitted emission lines.

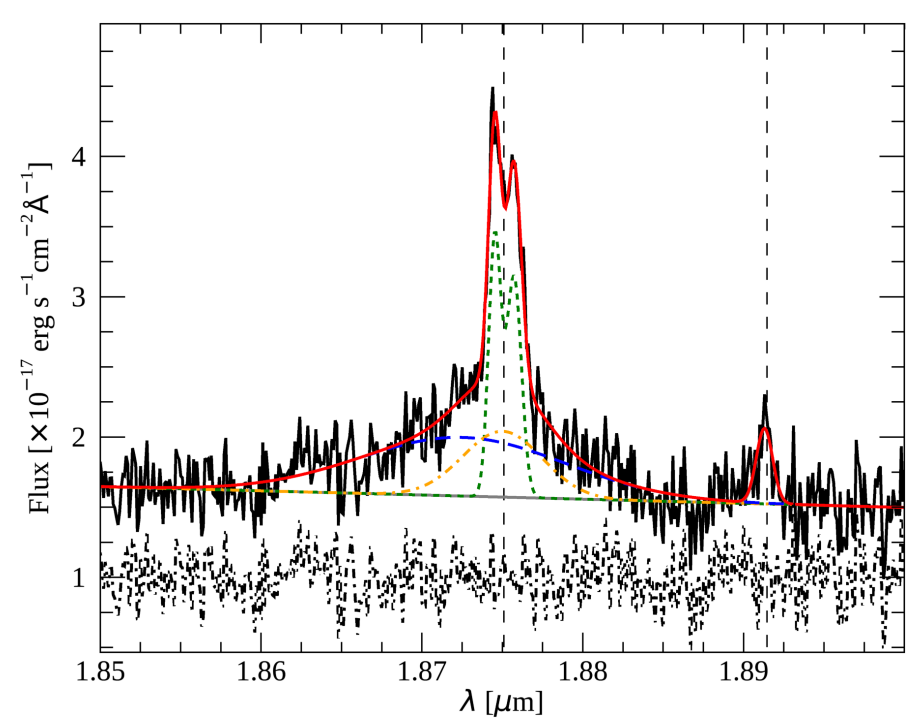

Fig. A.9. $\mathrm{Pa} \alpha$ and $\mathrm{H}_{2} \mathrm{~S}(4) 1-0$ emission lines (black solid line) and bestfit model (red solid line). The $\mathrm{Pa} \alpha$ line is modeled using the [O III] model, the $1 \mathrm{~N}$ and $2 \mathrm{~N}$ components are plotted together with the green dotted line, the $\mathrm{B}$ component is shown with the golden dot-dashed line, and the VB component with the blue dashed line. The $\mathrm{H}_{2} \mathrm{~S}(4) 1-0$ is modeled with a single Gaussian function indicated with the gray solid line. The residuals of the fit are normalized and plotted below the spectrum (black dot-dashed line). The vertical dashed lines mark the restframe wavelength of the fitted emission lines.

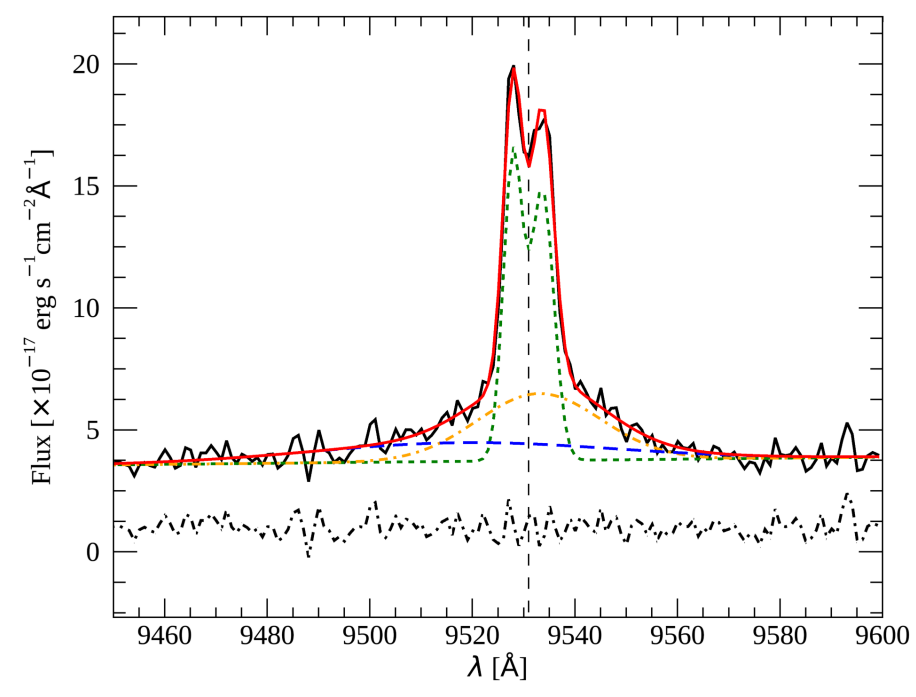

Fig. A.10. $[\mathrm{S}$ III $] \lambda 9531 \AA$ emission line (black solid line) and best-fit model (red solid line). The $[\mathrm{S}$ III] $] \lambda 9531 \AA$ line is modeled using the [O III] model, the $1 \mathrm{~N}$ and $2 \mathrm{~N}$ components are plotted together with the green dotted line, the I component is shown with the golden dot-dashed line, and the VB component with the blue dashed line. The residuals of the fit are normalized and plotted below the spectrum (black dot-dashed line). The vertical dashed lines mark the restframe wavelength of the fitted emission line. 

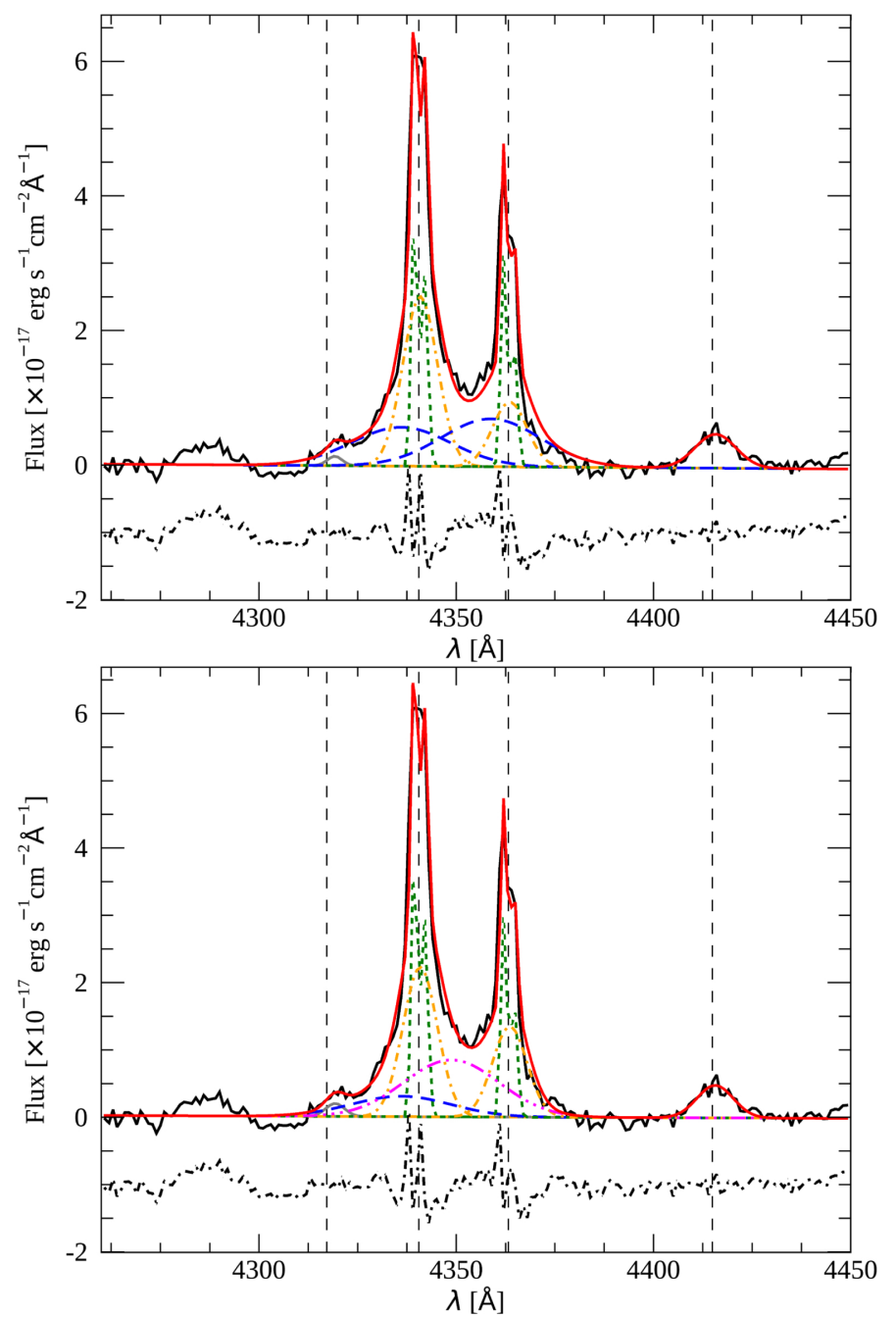

Fig. A.11. $\mathrm{H} \gamma+[\mathrm{O}$ III $] \lambda 4363 \AA$ emission lines (black solid line) and bestfit model (red solid line). The fitting procedure includes the modeling of two additional emission lines (gray solid line), most likely the $\left[\mathrm{O}_{\text {II }}\right] \lambda 4317.2 \AA$ and the $\left[\mathrm{O}_{\text {II }}\right] \lambda 4414.9 \AA$ lines, using a single Gaussian function. The residuals of the fit are normalized and plotted below the spectrum (black dot-dashed line). The vertical dashed lines mark the restframe wavelength of the fitted emission lines. The [O III] $] \lambda 4363 \AA$ line is modeled using the [O $\mathrm{III}]$ model, and the $\mathrm{H} \gamma$ line is modeled using both the [O $\mathrm{III}]$ model (top panel) and the $\mathrm{H} \beta$ model (bottom panel). The $1 \mathrm{~N}$ and $2 \mathrm{~N}$ components are plotted together with the green dotted line, the I component is shown with the golden dot-dashed line, and the VB component with the blue dashed line. The magenta triple dotdashed line indicates the broad redshifted component of the $\mathrm{H} \beta$ model.

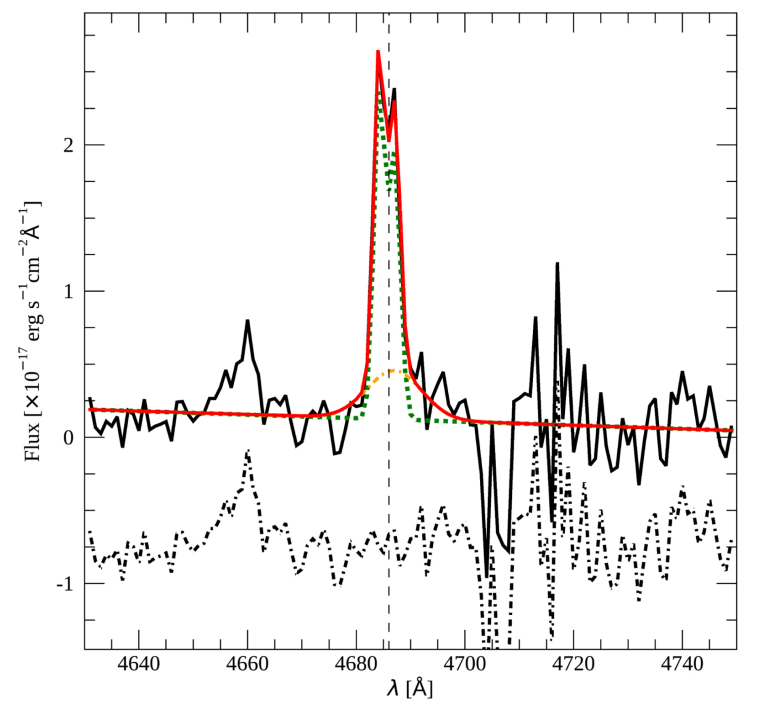

Fig. A.12. He iा $\lambda 4686 \AA$ emission line (black solid line) and best-fit model (red solid line). The line is modeled using the [O III] model, the $1 \mathrm{~N}$ and $2 \mathrm{~N}$ components are plotted together with the green dotted line, the I component is shown with the golden dot-dashed line. There is no evidence of a VB component. The residuals of the fit are normalized and plotted below the spectrum (black dot-dashed line). The vertical dashed lines mark the restframe wavelength of the fitted emission line.
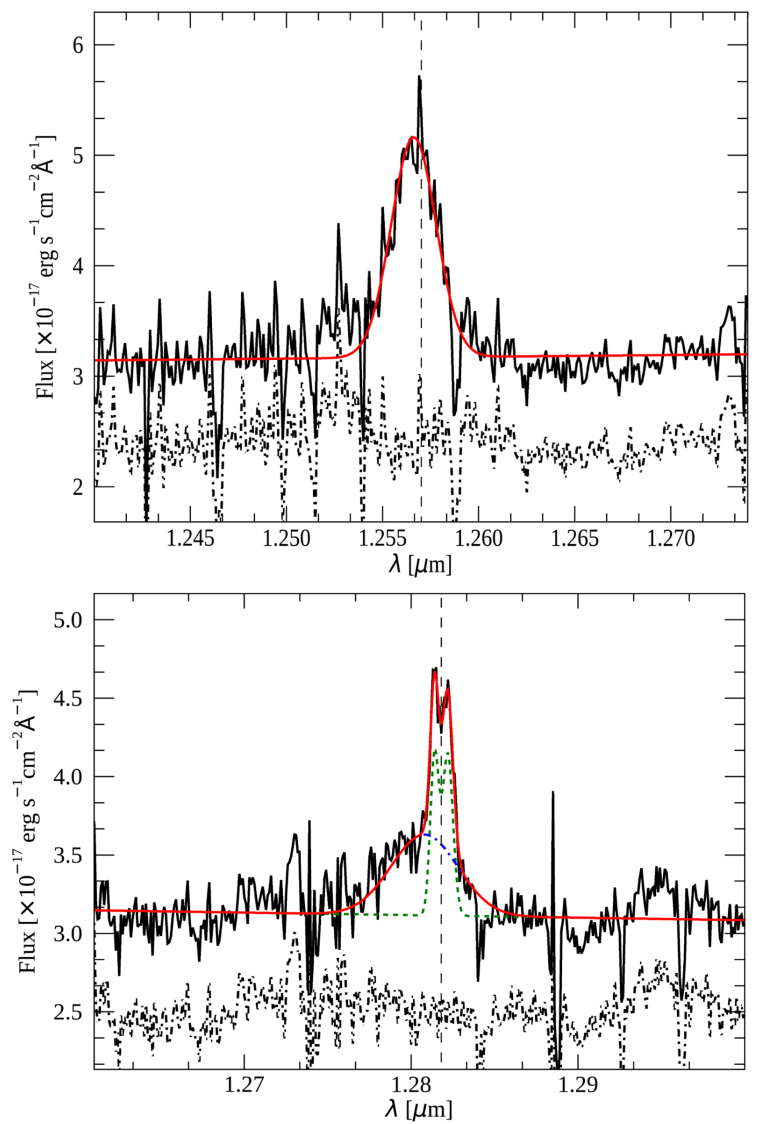

Fig. A.13. Section of the nuclear spectrum of PKS B1934-63 showing the $\mathrm{Fe}$ II $1.257 \mu \mathrm{m}$ (top panel) and the $\mathrm{Pa} \beta$ (bottom panel) emission lines (black solid line). The best-fit model is shown with the red solid line. The residuals of the fit are normalized and plotted below the spectrum (black dot-dashed line). The vertical dashed lines mark the restframe wavelength of the emission line. For the $\mathrm{Pa} \beta$, the model includes two narrow components (green dotted line) and a broad component (blue dashed line). 